\title{
Higgs boson mass and electroweak observables in the MRSSM
}

\author{
Philip Diessner, ${ }^{a}$ Jan Kalinowski, ${ }^{b}$ Wojciech Kotlarski ${ }^{b}$ and Dominik Stöckinger ${ }^{a}$ \\ ${ }^{a}$ Institut für Kern- und Teilchenphysik, Technische Universität Dresden, \\ 01069 Dresden, Germany \\ ${ }^{b}$ Faculty of Physics, University of Warsaw, \\ Pasteura 5, 02093 Warsaw, Poland \\ E-mail: philip.diessner@mailbox.tu-dresden.de, \\ jan.kalinowski@fuw.edu.pl, wojciech.kotlarski@fuw.edu.pl, \\ dominik.stoeckinger@tu-dresden.de
}

ABSTRACT: R-symmetry is a fundamental symmetry which can solve the SUSY flavor problem and relax the search limits on SUSY masses. Here we provide a complete next-toleading order computation and discussion of the lightest Higgs boson mass, the $\mathrm{W}$ boson mass and muon decay in the minimal R-symmetric SUSY model (MRSSM). This model contains non-MSSM particles including a Higgs triplet, Dirac gauginos and higgsinos, and leads to significant new tree-level and one-loop contributions to these observables. We show that the model can accommodate the measured values of the observables for interesting regions of parameter space with stop masses of order $1 \mathrm{TeV}$ in spite of the absence of stop mixing. We characterize these regions and provide typical benchmark points, which are also checked against further experimental constraints. A detailed exposition of the model, its mass matrices and its Feynman rules relevant for computations in this paper is also provided.

KEYWORDS: Supersymmetry Phenomenology

ArXIV EPRINT: 1410.4791 


\section{Contents}

1 Introduction $\quad 1$

2 Model definition 3

2.1 Field content, superpotential and soft masses 3

2.2 Tree-level Higgs boson masses $\quad 6$

2.3 Tree-level chargino and neutralino masses 9

$\begin{array}{lll}2.4 & \text { Calculation setup and benchmark points } & 10\end{array}$

3 Higgs boson mass prediction at one loop $\quad 11$

$\begin{array}{lll}3.1 & \text { Full one-loop calculation } & 12\end{array}$

$\begin{array}{lll}3.2 & \text { Effective potential approach } & 12\end{array}$

3.3 Comparison of different calculations and higher-order uncertainties 13

4 W boson mass prediction at one loop $\quad \mathbf{1 5}$

$\begin{array}{lll}4.1 & \text { Master formula for } m_{W} & 15\end{array}$

$\begin{array}{ll}4.2 \text { Computational framework } & 16\end{array}$

$\begin{array}{lll}4.3 & \text { Qualitative discussion } & 17\end{array}$

$\begin{array}{ll}4.4 \text { Comparison of full and approximate results } & 19\end{array}$

5 Numerical predictions $\quad 20$

5.1 Mass spectra 20

$\begin{array}{ll}5.2 & \text { Exploring the parameter space }\end{array}$

$\begin{array}{lll}5.3 & \text { Experimental constraints } & 25\end{array}$

6 Conclusions 26

$\begin{array}{ll}\text { A Feynman rules } & 27\end{array}$

B Calculation of vertex and box contributions to muon decay 29

\section{Introduction}

Supersymmetry is one of the most attractive concepts for physics beyond the Standard Model (SM). The Haag-Lopuszański-Sohnius theorem states that supersymmetry is the only possible non-trivial extension of the Poincaré algebra in a relativistic quantum field theory [1]. However, this theorem allows for a continuous R-symmetry [2, 3], an internal symmetry which does not commute with supersymmetry, but such a continuous Rsymmetry is not present in models such as the minimal supersymmetric standard model 
(MSSM). A continuous R-symmetry forbids Majorana gaugino masses, so it is phenomenologically only viable provided the gaugino masses are Dirac masses, which in turn implies that the gauge/gaugino sector is the one of an $N=2$ supersymmetric theory $[4,5]$, i.e. every gauge boson has a Dirac gaugino and a scalar superpartner. Properties of Dirac gauginos have already been extensively investigated independently of R-symmetry in the literature [6-9], in particular the required theory of supersymmetry breaking and $\beta$ functions have been developed [10-13].

Thus supersymmetry with R-symmetry is motivated and distinct from the MSSM since it contains a continuous R-symmetry, Dirac gauginos and an $N=2$ sector. A minimal viable model for this, the MRSSM, has been constructed in ref. [14]; for models with different R-charges see refs. [15-19]. Excitingly, the MRSSM alleviates some of the most important experimental constraints on the MSSM: the R-symmetry forbids large contributions to flavor-violating observables, so the MRSSM is generically in agreement with flavor data even for an anarchic flavor structure in the sfermion sector and for sfermion masses below the TeV scale [14, 20]. Similarly, heavy Dirac gluinos suppress the production cross section for squarks, making squarks below the TeV scale generically compatible with LHC data [21]. Furthermore, models with R-symmetry and/or Dirac gauginos contain promising dark matter candidates [22-24], and the collider physics of the extra, non-MSSMlike states has been studied [25-33].

In this paper we investigate a question of obvious importance after the Higgs boson discovery at the LHC: is the MRSSM compatible with the measured Higgs boson mass of $125 \mathrm{GeV}$, and what are the implications of this measurement on the MRSSM parameter space? The answer is not immediately obvious since at tree level the lightest Higgs boson mass is typically reduced compared to the MSSM, due to mixing with additional scalar states. Furthermore, an important enhancement mechanism in the MSSM - large stop mixing - is not available due to R-symmetry. Therefore, additional loop effects have to increase the Higgs boson mass.

The parameters entering the Higgs boson mass prediction also affect electroweak precision observables, in particular the $\mathrm{W}$ boson mass $m_{W}$. In addition, R-symmetry necessarily introduces an $\mathrm{SU}(2)$ scalar triplet, which can increase $m_{W}$ already at tree level. Hence we also study constraints on the model parameters and the Higgs boson mass from $m_{W}$. A similar set of questions has been studied recently in ref. [34], with the affirmative answer that the MRSSM is in agreement with the measured Higgs boson mass. Our study extends the one of that reference. We take into account the full one-loop corrections to both observables and perform extensive investigations of the parameter space. We define benchmark points illustrating different viable parameter regions and also verify that further theoretical and experimental constraints from Higgs, collider and low-energy physics are satisfied.

The following section presents the model in detail. All relevant mass and mixing matrices are provided, and the differences to the MSSM are explained. A complementary appendix A presents useful Feynman rules. Section 3 describes the computation of the Higgs boson mass and presents a comparison of the full one-loop calculation with an approximate result for the leading MRSSM-specific contributions. Even though stops do not mix in the MRSSM a $125 \mathrm{GeV}$ Higgs mass can be obtained with stop masses below $1 \mathrm{TeV}$. 


\begin{tabular}{|c|l|r||l|r|l|r|}
\hline \multicolumn{1}{|c}{ Field } & \multicolumn{2}{c}{ Superfield } & \multicolumn{2}{c|}{ Boson } & \multicolumn{2}{c|}{ Fermion } \\
\hline Gauge Vector & $\hat{g}, \hat{W}, \hat{B}$ & 0 & $g, W, B$ & 0 & $\tilde{g}, \tilde{W} \tilde{B}$ & +1 \\
Matter & $\hat{l}, \hat{e}$ & +1 & $\tilde{l}, \tilde{e}_{R}^{*}$ & +1 & $l, e_{R}^{*}$ & 0 \\
& $\hat{q}, \hat{d}, \hat{u}$ & +1 & $\tilde{q}, \tilde{d}_{R}^{*}, \tilde{u}_{R}^{*}$ & +1 & $q, d_{R}^{*}, u_{R}^{*}$ & 0 \\
$H$-Higgs & $\hat{H}_{d, u}$ & 0 & $H_{d, u}$ & 0 & $\tilde{H}_{d, u}$ & -1 \\
\hline R-Higgs & $\hat{R}_{d, u}$ & +2 & $R_{d, u}$ & +2 & $\tilde{R}_{d, u}$ & +1 \\
Adjoint Chiral & $\hat{\mathcal{O}}, \hat{T}, \hat{S}$ & 0 & $O, T, S$ & 0 & $\tilde{O}, \tilde{T}, \tilde{S}$ & -1 \\
\hline
\end{tabular}

Table 1. The R-charges of the superfields and the corresponding bosonic and fermionic components.

Section 4 provides a detailed discussion of the $\mathrm{W}$ boson mass and the leading contributions to the $T$-parameter; the details of the calculations are relegated to appendix B. Our main results are given in section 5. The parameter dependence of both observables is discussed, viable parameter regions are identified and the detailed predictions for a set of benchmark points are given.

\section{Model definition}

\subsection{Field content, superpotential and soft masses}

If we assign R-charges to the MSSM superfields in such a way that all Standard Model particles have $\mathrm{R}=0$ (in analogy to discrete $\mathrm{R}$-parity), Majorana gaugino, the $\mu$-term and higgsino mass terms are forbidden. Therefore, imposing R-symmetry on the MSSM requires an enlarged field content to account for non-vanishing gaugino and higgsino masses. The minimal R-symmetric extension of the supersymmetric model (MRSSM), which we will analyze, consists of the standard MSSM matter, Higgs and gauge superfields augmented by adjoint chiral superfields $\hat{\mathcal{O}}, \hat{T}, \hat{S}$ with R-charge 0 for each gauge sector, and two Higgs iso-doublet superfields $\hat{R}_{d, u}$ with R-charge 2 . The R-charges of the MRSSM superfields and their component fields are as listed in table 1 , if by convention the fermionic coordinates $\theta, \bar{\theta}$ have R-charge $+1,-1$.

With the above assignments the gauge, matter-gauge and Higgs-gauge actions of the MSSM are R-invariant, as well as the action associated with the trilinear Yukawa terms $Y_{d} \hat{d} \hat{q} \hat{H}_{d}$ etc in the superpotential. In contrast, the standard $\mu$ term is forbidden by the Rsymmetry. However, the presence of two iso-doublet superfields $\hat{R}_{u}$ and $\hat{R}_{d}$ with R-charge 2 together with the standard iso-doublets $\hat{H}_{u}$ and $\hat{H}_{d}$ allows to generate R-symmetric $\mu$-type terms and the corresponding higgsino masses,

$$
W \ni \mu_{d} \hat{R}_{d} \cdot \hat{H}_{d}+\mu_{u} \hat{R}_{u} \cdot \hat{H}_{u} .
$$

The appearance of the supersymmetric Higgs-higgsino mass term in eq. (2.1) can be thought of as arising from the R-symmetric operator involving the hidden sector F-type spurion $X=\theta^{2} F$

$$
\int d^{4} \theta\left(\frac{X^{\dagger}}{M} \hat{R}_{d} \cdot \hat{H}_{d}+\frac{X^{\dagger}}{M} \hat{R}_{u} \cdot \hat{H}_{u}\right) \ni \mu_{d} \hat{R}_{d} \cdot \hat{H}_{d}+\mu_{u} \hat{R}_{u} \cdot \hat{H}_{u}
$$


Similarly, the trilinear couplings of the electroweak $\hat{T}, \hat{S}$ adjoint chiral superfields to the Higgs doublets can be generated,

$$
W \ni \Lambda_{d} \hat{R}_{d} \cdot \hat{T} \hat{H}_{d}+\lambda_{d} \hat{S} \hat{R}_{d} \cdot \hat{H}_{d}+(d \rightarrow u)
$$

where the dot denotes $\epsilon$ contraction with $\epsilon_{12}=+1$ and where the triplet is defined as

$$
\hat{T}=\left(\begin{array}{cc}
\hat{T}_{0} / \sqrt{2} & \hat{T}_{+} \\
\hat{T}_{-} & -\hat{T}_{0} / \sqrt{2}
\end{array}\right)
$$

to have canonical kinetic terms. As we will see, these trilinear couplings will play an important role in obtaining a Higgs boson mass close to $125 \mathrm{GeV}$.

Thus the MRSSM superpotential takes the form of

$$
\begin{aligned}
W= & \mu_{d} \hat{R}_{d} \cdot \hat{H}_{d}+\mu_{u} \hat{R}_{u} \cdot \hat{H}_{u}+\Lambda_{d} \hat{R}_{d} \cdot \hat{T} \hat{H}_{d}+\Lambda_{u} \hat{R}_{u} \cdot \hat{T} \hat{H}_{u} \\
& +\lambda_{d} \hat{S} \hat{R}_{d} \cdot \hat{H}_{d}+\lambda_{u} \hat{S} \hat{R}_{u} \cdot \hat{H}_{u}-Y_{d} \hat{d} \hat{q} \cdot \hat{H}_{d}-Y_{e} \hat{e} \hat{l} \cdot \hat{H}_{d}+Y_{u} \hat{u} \hat{q} \cdot \hat{H}_{u} .
\end{aligned}
$$

Note that R-symmetry also forbids all baryon- and lepton-number violating terms in the superpotential (as well as dimension-five operators mediating proton decay) $[35,36]$ and that the R-symmetry is being preserved when the electroweak symmetry is broken.

Turning to soft breaking, the usual soft mass terms of the MSSM scalar fields are allowed just like in the MSSM. The MSSM A-terms, however, are zero due to R-symmetry. Importantly, soft Majorana gaugino mass terms are also forbidden by R-symmetry, but the fermionic components of the chiral adjoints, $\hat{\Phi}_{i}=\hat{\mathcal{O}}, \hat{T}, \hat{S}$ for each standard model gauge group $i=\mathrm{SU}(3), \mathrm{SU}(2), \mathrm{U}(1)$ respectively, can be paired with standard gauginos $\tilde{g}, \tilde{W}, \tilde{B}$ to build Dirac fermions and the corresponding mass terms. The Dirac masses for the gauginos can be considered as elements of a general supersymmetric theory broken softly by hidden sector spurions $W_{\alpha}^{\prime}=\theta_{\alpha} D$ from a hidden sector $\mathrm{U}(1)^{\prime}$ that acquires a D-term. The Dirac gaugino mass is generated from the R-symmetric operator involving the D-type spurion [8],

$$
\int d^{2} \theta \frac{\hat{W}_{\alpha}^{\prime}}{M} W_{i}^{\alpha} \hat{\Phi}_{i} \ni M_{i}^{D} \tilde{g}_{i} \tilde{g}_{i}^{\prime}
$$

where $\mathrm{M}$ is the mediation scale of SUSY breaking from the hidden sector to the visible sector, $W_{i}^{\alpha}$ represents the gauge superfield strength tensors, $\tilde{g}_{i}=\tilde{g}, \tilde{W}, \tilde{B}$ is the gaugino, and $\tilde{g}_{i}^{\prime}=\tilde{O}, \tilde{T}, \tilde{S}$ is the corresponding Dirac partner with opposite R-charge. Note that integrating out the spurion in eq. (2.6) generates additional terms with the D-fields and the scalar components, which leads to the appearance of Dirac masses in the scalar sector as well,

$$
V_{D}=M_{B}^{D}\left(\tilde{B} \tilde{S}-\sqrt{2} \mathcal{D}_{B} S\right)+M_{W}^{D}\left(\tilde{W}^{a} \tilde{T}^{a}-\sqrt{2} \mathcal{D}_{W}^{a} T^{a}\right)+M_{g}^{D}\left(\tilde{g}^{a} \tilde{O}^{a}-\sqrt{2} \mathcal{D}_{g}^{a} O^{a}\right)+\text { h.c. }
$$


In a similar fashion, the soft SUSY breaking $B_{\mu}$, the Higgs and R-Higgs scalar masses can be generated by R-symmetric interactions with the hidden sector spurion $X$

$$
\begin{aligned}
& \int d^{4} \theta \frac{X^{\dagger} \hat{X}}{M^{2}} \hat{H}_{d} \cdot \hat{H}_{u} \ni B_{\mu} H_{d} \cdot H_{u}, \\
& \int d^{4} \theta \frac{X^{\dagger} \hat{X}}{M^{2}} \hat{R}_{d}^{\dagger} \hat{R}_{d} \ni m_{R_{d}}^{2}\left(\left|R_{d}^{+}\right|^{2}+\left|R_{d}^{0}\right|^{2}\right), \text { and } d \rightarrow u,
\end{aligned}
$$

as well as the SUSY breaking adjoint scalar mass terms can be generated $\left(\Phi_{i}=O, T, S\right)$

$$
\int d^{4} \theta \frac{X^{\dagger} \hat{X}^{2}}{M^{2}} \hat{\Phi}_{i}^{\dagger} \hat{\Phi}_{i} \ni m_{i}^{2}\left|\Phi_{i}\right|^{2}
$$

suggesting that $\mu_{d, u}, B_{\mu}$ and soft scalar masses could be of the same order, i.e. of the order of SUSY breaking scale. Note that the bilinear coupling of the R-Higgs fields, like in eq. (2.8), has R-charge 4 and therefore is forbidden in the R-symmetric theory. Thus only the $B_{\mu}$ term destroys the exchange symmetry between the $H$ and R-Higgs fields.

For completeness, it should be noticed that bilinear couplings of the $\hat{X}$ fields with the adjoint chiral fields can also generate contributions

$$
\int d^{4} \theta \frac{X^{\dagger} \hat{X}}{M^{2}} \operatorname{Tr}\left[\hat{\Phi}_{i} \hat{\Phi}_{i}\right] \ni B_{i} \Phi_{i} \Phi_{i}
$$

to the (holomorphic) soft masses of the adjoint scalars in addition to the above soft terms. Such terms split scalar and pseudoscalar masses squared by equal amounts. If the pseudoscalar gets a negative contribution to its mass squared, it may generate color- or chargebreaking minima. Although there is no symmetry that allows non-holomorphic (2.10), but forbids holomorphic (2.11) mass terms, these terms are technically independent [8]. In the following we will not study theoretical points such as SUSY breaking mechanisms, but explore only the phenomenology of the R-symmetric low-energy theory. Therefore, for simplicity we will neglect holomorphic mass terms for $S$ and $T$ adjoint scalars, as well as their trilinear couplings among themselves and to the Higgs bosons since their presence does not influence our results significantly (however, see also [34]). Thus the soft-breaking scalar mass terms read

$$
\begin{aligned}
V_{S B}^{E W}= & B_{\mu}\left(H_{d}^{-} H_{u}^{+}-H_{d}^{0} H_{u}^{0}\right)+\text { h.c. } \\
& +m_{H_{d}}^{2}\left(\left|H_{d}^{0}\right|^{2}+\left|H_{d}^{-}\right|^{2}\right)+m_{H_{u}}^{2}\left(\left|H_{u}^{0}\right|^{2}+\left|H_{u}^{+}\right|^{2}\right) \\
& +m_{R_{d}}^{2}\left(\left|R_{d}^{0}\right|^{2}+\left|R_{d}^{+}\right|^{2}\right)+m_{R_{u}}^{2}\left|R_{u}^{0}\right|^{2}+m_{R_{u}}^{2}\left|R_{d}^{-}\right|^{2} \\
& +m_{S}^{2}|S|^{2}+m_{T}^{2}\left|T^{0}\right|^{2}+m_{T}^{2}\left|T^{-}\right|^{2}+m_{T}^{2}\left|T^{+}\right|^{2}+m_{O}^{2}|O|^{2} \\
& +\tilde{d}_{L, i}^{*} m_{q, i j}^{2} \tilde{d}_{L, j}+\tilde{d}_{R, i}^{*} m_{d, i j}^{2} \tilde{d}_{R, j}+\tilde{u}_{L, i}^{*} m_{q, i j}^{2} \tilde{u}_{L, j}+\tilde{u}_{R, i}^{*} m_{u, i j}^{2} \tilde{u}_{R, j} \\
& +\tilde{e}_{L, i}^{*} m_{l, i j}^{2} \tilde{e}_{L, j}+\tilde{e}_{R, i}^{*} m_{e, i j}^{2} \tilde{e}_{R, j}+\tilde{\nu}_{L, i}^{*} m_{l, i j}^{2} \tilde{\nu}_{L, j} .
\end{aligned}
$$

Note that R-symmetry forbids all trilinear scalar couplings involving Higgs bosons to squarks and sleptons, which in the MSSM are notoriously unwanted sources of flavor violation. Since in this paper we do not discuss lepton flavor violating phenomena, for simplicity we assume that slepton mass matrices are diagonal. 


\subsection{Tree-level Higgs boson masses}

The electroweak part of the tree-level scalar potential for the neutral fields takes the form of

$$
\begin{aligned}
& V^{E W}=\left(m_{H_{d}}^{2}+\mu_{d}^{2}\right)\left|H_{d}^{0}\right|^{2}+\left(m_{H_{u}}^{2}+\mu_{u}^{2}\right)\left|H_{u}^{0}\right|^{2}-B_{\mu}\left(H_{d}^{0} H_{u}^{0}+\text { h.c. }\right) \\
& +\left(m_{R_{d}}^{2}+\mu_{d}^{2}\right)\left|R_{d}^{0}\right|^{2}+\left(m_{R_{u}}^{2}+\mu_{u}^{2}\right)\left|R_{u}^{0}\right|^{2}+m_{T}^{2}\left|T^{0}\right|^{2}+m_{S}^{2}|S|^{2} \\
& +\left(2 M_{W}^{D} \Re\left(T^{0}\right)\right)^{2}-\left(2 M_{B}^{D} \Re(S)\right)^{2}+\frac{1}{8}\left(g_{1}^{2}+g_{2}^{2}\right)\left(\left|H_{d}^{0}\right|^{2}-\left|H_{u}^{0}\right|^{2}-\left|R_{d}^{0}\right|^{2}+\left|R_{u}^{0}\right|^{2}\right)^{2} \\
& -\left[\left(g_{1} M_{B}^{D}-\sqrt{2} \lambda_{d} \mu_{d}\right) \sqrt{2} \Re(S)-\left(g_{2} M_{W}^{D}+\Lambda_{d} \mu_{d}\right) \sqrt{2} \Re\left(T^{0}\right)-\left|\lambda_{d} S+\frac{1}{\sqrt{2}} \Lambda_{d} T^{0}\right|^{2}\right]\left|H_{d}^{0}\right|^{2} \\
& +\left[\left(g_{1} M_{B}^{D}-\sqrt{2} \lambda_{u} \mu_{u}\right) \sqrt{2} \Re(S)-\left(g_{2} M_{W}^{D}+\Lambda_{u} \mu_{u}\right) \sqrt{2} \Re\left(T^{0}\right)+\left|\lambda_{u} S-\frac{1}{\sqrt{2}} \Lambda_{u} T^{0}\right|^{2}\right]\left|H_{u}^{0}\right|^{2} \\
& +\left[\left(g_{1} M_{B}^{D}+\sqrt{2} \lambda_{d} \mu_{d}\right) \sqrt{2} \Re(S)-\left(g_{2} M_{W}^{D}-\Lambda_{d} \mu_{d}\right) \sqrt{2} \Re\left(T^{0}\right)+\left|\lambda_{d} S+\frac{1}{\sqrt{2}} \Lambda_{d} T^{0}\right|^{2}\right]\left|R_{d}^{0}\right|^{2} \\
& -\left[\left(g_{1} M_{B}^{D}-\sqrt{2} \lambda_{u} \mu_{u}\right) \sqrt{2} \Re(S)-\left(g_{2} M_{W}^{D}-\Lambda_{u} \mu_{u}\right) \sqrt{2} \Re\left(T^{0}\right)-\left|\lambda_{u} S-\frac{1}{\sqrt{2}} \Lambda_{u} T^{0}\right|^{2}\right]\left|R_{u}^{0}\right|^{2} \\
& +\left(\lambda_{d}^{2}+\frac{1}{2} \Lambda_{d}\right)\left|H_{d}^{0} R_{d}^{0}\right|^{2}+\left(\lambda_{u}^{2}+\frac{1}{2} \Lambda_{u}^{2}\right)\left|H_{u}^{0} R_{u}^{0}\right|^{2}-\left(\lambda_{d} \lambda_{u}-\frac{1}{2} \Lambda_{d} \Lambda_{u}\right)\left(H_{d}^{0} R_{d}^{0} H_{u}^{0 \dagger} R_{u}^{0 \dagger}+\text { h.c. }\right)
\end{aligned}
$$

Since R-Higgs bosons carry R-charge 2, they do not develop vacuum expectation values. Therefore, for electroweak symmetry breaking (EWSB) we write the $R=0$ neutral EW scalars as

$$
\begin{aligned}
H_{d}^{0} & =\frac{1}{\sqrt{2}}\left(v_{d}+\phi_{d}+i \sigma_{d}\right), & H_{u}^{0} & =\frac{1}{\sqrt{2}}\left(v_{u}+\phi_{u}+i \sigma_{u}\right), \\
T^{0} & =\frac{1}{\sqrt{2}}\left(v_{T}+\phi_{T}+i \sigma_{T}\right), & S & =\frac{1}{\sqrt{2}}\left(v_{S}+\phi_{S}+i \sigma_{S}\right) .
\end{aligned}
$$

After EWSB the singlet and triplet vacuum expectation values effectively modify the $\mu$ parameters of the model, and it is useful to define the abbreviations

$$
\mu_{i}^{\mathrm{eff}, \pm}=\mu_{i}+\frac{\lambda_{i} v_{S}}{\sqrt{2}} \pm \frac{\Lambda_{i} v_{T}}{2}, \quad \quad \mu_{i}^{\mathrm{eff}, 0}=\mu_{i}+\frac{\lambda_{i} v_{S}}{\sqrt{2}}, \quad i=u, d .
$$

The minimization conditions for the scalar potential, or tadpole equations, then read

$$
0=t_{d}=t_{u}=t_{T}=t_{S},
$$

where the tree-level tadpoles $t_{i} \equiv \frac{\partial V^{E W}}{\partial \phi_{i}}$ are

$$
\begin{aligned}
t_{d} & =v_{d}\left[\frac{1}{8}\left(g_{1}^{2}+g_{2}^{2}\right)\left(v_{d}^{2}-v_{u}^{2}\right)-g_{1} M_{B}^{D} v_{S}+g_{2} M_{W}^{D} v_{T}+m_{H_{d}}^{2}+\left(\mu_{d}^{\text {eff, }+}\right)^{2}\right]-v_{u} B_{\mu}, \\
t_{u} & =v_{u}\left[\frac{1}{8}\left(g_{1}^{2}+g_{2}^{2}\right)\left(v_{u}^{2}-v_{d}^{2}\right)+g_{1} M_{B}^{D} v_{S}-g_{2} M_{W}^{D} v_{T}+m_{H_{u}}^{2}+\left(\mu_{u}^{\mathrm{eff},-}\right)^{2}\right]-v_{d} B_{\mu}, \\
t_{T} & =\frac{1}{2} g_{2} M_{W}^{D}\left(v_{d}^{2}-v_{u}^{2}\right)+\frac{1}{2} \Lambda_{d} v_{d}^{2} \mu_{d}^{\mathrm{eff},+}-\Lambda_{u} v_{u}^{2} \mu_{u}^{\mathrm{eff},-}+4\left(M_{W}^{D}\right)^{2} v_{T}+m_{T}^{2} v_{T}, \\
t_{S} & =\frac{1}{2} g_{1} M_{B}^{D}\left(v_{u}^{2}-v_{d}^{2}\right)+\frac{1}{\sqrt{2}} \lambda_{d} v_{d}^{2} \mu_{d}^{\mathrm{eff},+}+\lambda_{u} v_{u}^{2} \mu_{u}^{\text {eff, }-}+4\left(M_{B}^{D}\right)^{2} v_{S}+m_{S}^{2} v_{S} .
\end{aligned}
$$

The tadpole eqs. (2.15) are used to substitute $m_{H_{d}}^{2}$ and $m_{H_{u}}^{2}$ as usual with $v_{d}$ and $v_{u}$, introducing $v=\sqrt{v_{u}^{2}+v_{d}^{2}}$ and $\tan \beta=v_{u} / v_{d}$ as in the MSSM. The other two equations are 
solved for $v_{T}$ and $v_{S}$, allowing us to use $m_{S}^{2}$ and $m_{T}^{2}$ as input parameters. This can be done analytically, but the expressions have no simple form and will not be written here. Because of this, we will use those equations in the following analytical analyses only in cases where they simplify the expressions significantly, as they do in the case of the pseudo-scalar Higgs mass matrix, and only to some extent in the case of the scalar Higgs one. In other cases, the appearing $v_{T}$ and $v_{S}$ are always functions of the other model parameters and this is taken into account in numerical calculations.

Masses for the gauge bosons arise in the usual form for the $\mathrm{Z}$ boson, but the $\mathrm{W}$ boson has a contribution from the triplet vev:

$$
m_{Z}^{2}=\frac{g_{1}^{2}+g_{2}^{2}}{4} v^{2}, \quad m_{W}^{2}=\frac{g_{2}^{2}}{4} v^{2}+g_{2}^{2} v_{T}^{2}, \quad \hat{\rho}_{\text {tree }}=1+\frac{4 v_{T}^{2}}{v^{2}} .
$$

This will shift the $\rho$ parameter away from one already at the tree level and gives an upper limit on $\left|v_{T}\right| \lesssim 4 \mathrm{GeV}$, which is taken into account when studying parameter points. Further consequences at higher orders will be investigated in section 4 .

Using the tadpole eqs. (2.16), the pseudo-scalar Higgs boson mass matrix in the basis $\left(\sigma_{d}, \sigma_{u}, \sigma_{S}, \sigma_{T}\right)$ has a simple form (in Landau gauge)

$$
\mathcal{M}_{A}=\left(\begin{array}{cccc}
B_{\mu} \frac{v_{u}}{v_{d}} & B_{\mu} & 0 & 0 \\
B_{\mu} & B_{\mu} \frac{v_{d}}{v_{u}} & 0 & 0 \\
0 & 0 & m_{S}^{2}+\frac{\lambda_{d}^{2} v_{d}^{2}+\lambda_{u}^{2} v_{u}^{2}}{2} & \frac{\lambda_{d} \Lambda_{d} v_{d}^{2}-\lambda_{u} \Lambda_{u} v_{u}^{2}}{2 \sqrt{2}} \\
0 & 0 & \frac{\lambda_{d} \Lambda_{d} v_{d}^{2}-\lambda_{u} \Lambda_{u} v_{u}^{2}}{2 \sqrt{2}} & m_{T}^{2}+\frac{\Lambda_{d}^{2} v_{d}^{2}+\Lambda_{u}^{2} v_{u}^{2}}{4}
\end{array}\right) .
$$

Due to the absence of mixing terms, the MSSM-like upper left and the singlet-triplet bottom right sub matrices are independent of each other. Therefore, the neutral Goldstone boson and one of the pseudo-scalar Higgs bosons with $m_{A}^{2}=2 B_{\mu} / \sin 2 \beta$ will be as in the MSSM while the other two are singlet- and triplet-like. To ensure that EWSB occurs correctly, meaning no tachyons arise when diagonalizing the mass matrices, we restrict the values of $m_{S}^{2}$ and $m_{T}^{2}$ to always being positive.

The scalar Higgs boson mass matrix in the weak basis $\left(\phi_{d}, \phi_{u}, \phi_{S}, \phi_{T}\right)$ is given by

$$
\mathcal{M}_{H^{0}}=\left(\begin{array}{cc}
\mathcal{M}_{\mathrm{MSSM}} & \mathcal{M}_{21}^{T} \\
\mathcal{M}_{21} & \mathcal{M}_{22}
\end{array}\right)
$$

with the sub-matrices $\left(c_{\beta}=\cos \beta, s_{\beta}=\sin \beta\right.$, etc $)$

$$
\begin{aligned}
\mathcal{M}_{\mathrm{MSSM}}= & \left(\begin{array}{cc}
m_{Z}^{2} c_{\beta}^{2}+m_{A}^{2} s_{\beta}^{2} & -\left(m_{Z}^{2}+m_{A}^{2}\right) s_{\beta} c_{\beta} \\
-\left(m_{Z}^{2}+m_{A}^{2}\right) s_{\beta} c_{\beta} & m_{Z}^{2} s_{\beta}^{2}+m_{A}^{2} c_{\beta}^{2}
\end{array}\right), \\
\mathcal{M}_{22}= & \left(\begin{array}{cc}
4\left(M_{B}^{D}\right)^{2}+m_{S}^{2}+\frac{\lambda_{d}^{2} v_{d}^{2}+\lambda_{u}^{2} v_{u}^{2}}{2} & \frac{\lambda_{d} \Lambda_{d} v_{d}^{2}-\lambda_{u} \Lambda_{u} v_{u}^{2}}{2 \sqrt{2}} \\
\frac{\lambda_{d} \Lambda_{d} v_{d}^{2}-\lambda_{u} \Lambda_{u} v_{u}^{2}}{2 \sqrt{2}} & 4\left(M_{W}^{D}\right)^{2}+m_{T}^{2}+\frac{\Lambda_{d}^{2} v_{d}^{2}+\Lambda_{u}^{2} v_{u}^{2}}{4}
\end{array}\right), \\
\mathcal{M}_{21}= & \left(\begin{array}{cc}
v_{d}\left(\sqrt{2} \lambda_{d} \mu_{d}^{\mathrm{eff},+}-g_{1} M_{B}^{D}\right) & v_{u}\left(\sqrt{2} \lambda_{u} \mu_{u}^{\mathrm{eff},-}+g_{1} M_{B}^{D}\right) \\
v_{d}\left(\Lambda_{d} \mu_{d}^{\mathrm{eff},+}+g_{2} M_{W}^{D}\right) & -v_{u}\left(\Lambda_{u} \mu_{u}^{\mathrm{eff},-}+g_{2} M_{W}^{D}\right)
\end{array}\right) .
\end{aligned}
$$


Again, $\mathcal{M}_{\text {MSSM }}$ is MSSM-like and $\mathcal{M}_{22}$ singlet-triplet-like, but compared to the pseudoscalar case, additional mixing exists through $\mathcal{M}_{21}$. Generally, this will lead to a reduction of the lightest Higgs boson mass at tree-level compared to the MSSM. An approximate formula for the reduction can be given for the lightest Higgs boson mass when using the MSSM mixing angle $\alpha$ to diagonalize $\mathcal{M}_{\text {MSSM }}$ for large $m_{A}^{2}$ when $\alpha=\beta-\pi / 2$, further assuming $\lambda=\lambda_{u}=-\lambda_{d}, \Lambda=\Lambda_{u}=\Lambda_{d}, \mu_{u}=\mu_{d}=\mu$ and $v_{S} \approx v_{T} \approx 0$ :

$$
m_{H_{1}, \text { approx }}^{2}=m_{Z}^{2} \cos ^{2} 2 \beta-v^{2}\left(\frac{\left(g_{1} M_{B}^{D}+\sqrt{2} \lambda \mu\right)^{2}}{4\left(M_{B}^{D}\right)^{2}+m_{S}^{2}}+\frac{\left(g_{2} M_{W}^{D}+\Lambda \mu\right)^{2}}{4\left(M_{W}^{D}\right)^{2}+m_{T}^{2}}\right) \cos ^{2} 2 \beta .
$$

It can be seen that the upper limit of the MSSM at the tree-level is reduced by terms depending on the new model parameters. Thus one-loop contributions are important, and a detailed study will be presented in section 3 .

The charged Higgs boson mass matrix in the weak basis $\left(H_{d}^{-*}, H_{u}^{+}, T^{-*}, T^{+}\right)$is given as

$$
\mathcal{M}_{H^{ \pm}}=\left(\begin{array}{cc}
\mathcal{M}_{\mathrm{MSSM}, \pm} & \mathcal{M}_{21, \pm}^{T} \\
\mathcal{M}_{21, \pm} & \mathcal{M}_{22, \pm}
\end{array}\right)
$$

with the sub-matrices

$$
\begin{aligned}
\mathcal{M}_{\mathrm{MSSM}, \pm}= & \left(\begin{array}{cc}
m_{H^{ \pm}}^{2} c_{\beta}^{2}-2 v_{T}\left(g_{2} M_{W}^{D}+\Lambda_{d} \mu_{d}^{\mathrm{eff}, 0}\right) & m_{H^{ \pm}}^{2} s_{\beta} c_{\beta} \\
m_{H^{ \pm}}^{2} s_{\beta} c_{\beta} & m_{H^{ \pm}}^{2} c_{\beta}^{2}+2 v_{T}\left(g_{2} M_{W}^{D}+\Lambda_{u} \mu_{u}^{\mathrm{eff}, 0}\right)
\end{array}\right) \\
\mathcal{M}_{22, \pm}= & \left(\begin{array}{cc}
2\left(M_{W}^{D}\right)^{2}+m_{T}^{2} & 2\left(M_{W}^{D}\right)^{2} \\
2\left(M_{W}^{D}\right)^{2} & 2\left(M_{W}^{D}\right)^{2}+m_{T}^{2}
\end{array}\right) \\
& +\left(\begin{array}{cc}
\frac{g_{2}^{2} v_{T}^{2}+\Lambda_{d}^{2} v^{2} c_{\beta}^{2}}{2}-\frac{g_{2}^{2} v^{2} \cos 2 \beta}{4} & -\frac{g_{2}^{2} v_{T}^{2}}{2} \\
-\frac{g_{2}^{2} v_{T}^{2}}{2} & \frac{g_{2}^{2} v_{T}^{2}+\Lambda_{u}^{2} v^{2} s_{\beta}^{2}}{2}+\frac{g_{2}^{2} v^{2} \cos 2 \beta}{4}
\end{array}\right) \\
\mathcal{M}_{21, \pm}= & \left(\begin{array}{cc}
\frac{v_{d}}{2 \sqrt{2}}\left(2 \Lambda_{d} \mu_{d}^{\mathrm{eff},-}+2 g_{2} M_{W}^{D}+v_{T} g_{2}^{2}\right) & \frac{v_{u}}{2 \sqrt{2}}\left(2 \Lambda_{u} \mu_{u}^{\mathrm{eff},-}+2 g_{2} M_{W}^{D}+v_{T} g_{2}^{2}\right) \\
\frac{v_{d}}{2 \sqrt{2}}\left(2 \Lambda_{d} \mu_{d}^{\mathrm{eff},+}+2 g_{2} M_{W}^{D}-v_{T} g_{2}^{2}\right) & \frac{v_{u}}{2 \sqrt{2}}\left(2 \Lambda_{u} \mu_{u}^{\mathrm{eff},+}+2 g_{2} M_{W}^{D}-v_{T} g_{2}^{2}\right)
\end{array}\right)
\end{aligned}
$$

where the charged Higgs mass parameter $m_{H^{ \pm}}^{2}=m_{A}^{2}+\frac{g_{2}^{2} v^{2}}{4}$ reads as in the MSSM.

The mass matrix of neutral R-Higgs bosons is

$$
\mathcal{M}_{R}=\left(\begin{array}{cc}
m_{R_{d} R_{d}}^{2} & \frac{1}{4}\left(\Lambda_{u} \Lambda_{d}-2 \lambda_{u} \lambda_{d}\right) v_{u} v_{d} \\
\frac{1}{4}\left(\Lambda_{u} \Lambda_{d}-2 \lambda_{u} \lambda_{d}\right) v_{u} v_{d} & m_{R_{u} R_{u}}^{2}
\end{array}\right)
$$

with

$$
\begin{aligned}
& m_{R_{d} R_{d}}^{2}=m_{R_{d}}^{2}+\left(\mu_{d}^{\mathrm{eff},+}\right)^{2}+g_{1} M_{B}^{D} v_{S}-g_{2} M_{W}^{D} v_{T}+\frac{1}{8}\left[\left(g_{1}^{2}+g_{2}^{2}\right)\left(v_{u}^{2}-v_{d}^{2}\right)+4 \lambda_{d}^{2} v_{d}^{2}+2 \Lambda_{d}^{2} v_{d}^{2}\right], \\
& m_{R_{u} R_{u}}^{2}=m_{R_{u}}^{2}+\left(\mu_{u}^{\mathrm{eff},-}\right)^{2}-g_{1} M_{B}^{D} v_{S}+g_{2} M_{W}^{D} v_{T}+\frac{1}{8}\left[\left(g_{1}^{2}+g_{2}^{2}\right)\left(v_{d}^{2}-v_{u}^{2}\right)+4 \lambda_{u}^{2} v_{u}^{2}+2 \Lambda_{u}^{2} v_{u}^{2}\right] .
\end{aligned}
$$

It is diagonalized by an orthogonal matrix with mixing angle $\theta_{R}$.

Because of the R-symmetry the charged R-Higgs bosons do not mix and the mass eigenvalues are given as

$$
\begin{aligned}
& m_{R_{1}^{+}}^{2}=m_{R_{d}}^{2}+\left(\mu_{d}^{\mathrm{eff},-}\right)^{2}+g_{1} M_{B}^{D} v_{S}+g_{2} M_{W}^{D} v_{T}+\frac{1}{8}\left[\left(g_{1}^{2}-g_{2}^{2}\right)\left(v_{u}^{2}-v_{d}^{2}\right)+4 \Lambda_{d}^{2} v_{d}^{2}\right] \\
& m_{R_{2}^{+}}^{2}=m_{R_{u}}^{2}+\left(\mu_{u}^{\mathrm{eff},+}\right)^{2}-g_{1} M_{B}^{D} v_{S}-g_{2} M_{W}^{D} v_{T}+\frac{1}{8}\left[\left(g_{1}^{2}-g_{2}^{2}\right)\left(v_{d}^{2}-v_{u}^{2}\right)+4 \Lambda_{u}^{2} v_{u}^{2}\right] .
\end{aligned}
$$




\subsection{Tree-level chargino and neutralino masses}

For completeness and to fix the notation, we give below expressions for tree-level masses and mixing matrices of the charginos and neutralinos.

In the weak basis of eight neutral electroweak two-component fermions: $\xi_{i}=$ $\left(\tilde{B}, \tilde{W}^{0}, \tilde{R}_{d}^{0}, \tilde{R}_{u}^{0}\right), \zeta_{i}=\left(\tilde{S}, \tilde{T}^{0}, \tilde{H}_{d}^{0}, \tilde{H}_{u}^{0}\right)$ with R-charges $+1,-1$ respectively, the neutralino mass matrix takes the form of

$$
m_{\chi}=\left(\begin{array}{cccc}
M_{B}^{D} & 0 & -\frac{1}{2} g_{1} v_{d} & \frac{1}{2} g_{1} v_{u} \\
0 & M_{W}^{D} & \frac{1}{2} g_{2} v_{d} & -\frac{1}{2} g_{2} v_{u} \\
-\frac{1}{\sqrt{2}} \lambda_{d} v_{d} & -\frac{1}{2} \Lambda_{d} v_{d} & -\mu_{d}^{\text {eff, }+} & 0 \\
\frac{1}{\sqrt{2}} \lambda_{u} v_{u} & -\frac{1}{2} \Lambda_{u} v_{u} & 0 & \mu_{u}^{\text {eff,- }}
\end{array}\right) .
$$

The transformation to a diagonal mass matrix and mass eigenstates $\kappa_{i}$ and $\psi_{i}$ is performed by two unitary mixing matrices $N^{1}$ and $N^{2}$ as

$$
N^{1, *} m_{\chi} N^{2, \dagger}=m_{\chi}^{\text {diag }}, \quad \xi_{i}=\sum_{j=1}^{4} N_{j i}^{1, *} \kappa_{j}, \quad \zeta_{i}=\sum_{j=1}^{4} N_{i j}^{2, *} \psi_{j},
$$

and physical four-component Dirac neutralinos are constructed as

$$
\chi_{i}=\left(\begin{array}{c}
\kappa_{i} \\
\psi_{i}^{*}
\end{array}\right) \quad i=1,2,3,4 .
$$

The mass matrix of charginos in the weak basis of eight charged two-component fermions breaks into two $(2 \times 2)$ submatrices. The first, in the basis $\left(\tilde{T}^{-}, \tilde{H}_{d}^{-}\right),\left(\tilde{W}^{+}, \tilde{R}_{d}^{+}\right)$ of spinors with R-charge equal to electric charge, takes the form of

$$
m_{\chi^{+}}=\left(\begin{array}{cc}
g_{2} v_{T}+M_{W}^{D} & \frac{1}{\sqrt{2}} \Lambda_{d} v_{d} \\
\frac{1}{\sqrt{2}} g_{2} v_{d} & +\mu_{d}^{\text {eff, }-}
\end{array}\right)
$$

The diagonalization and transformation to mass eigenstates $\lambda_{i}^{ \pm}$is performed by two unitary matrices $U^{1}$ and $V^{1}$ as

$$
\begin{array}{rlrl}
U^{1, *} m_{\chi^{+}} V^{1, \dagger}=m_{\chi^{+}}^{\operatorname{diag}}, & \tilde{T}^{-}=\sum_{j=1}^{2} U_{j 1}^{1, *} \lambda_{j}^{-}, & \tilde{H}_{d}^{-}=\sum_{j=1}^{2} U_{j 2}^{1, *} \lambda_{j}^{-}, \\
\tilde{W}^{+}=\sum_{j=1}^{2} V_{1 j}^{1, *} \lambda_{j}^{+}, & R_{d}^{+}=\sum_{j=1}^{2} V_{2 j}^{1, *} \lambda_{j}^{+}
\end{array}
$$

and the corresponding physical four-component charginos are built as

$$
\chi_{i}^{+}=\left(\begin{array}{c}
\lambda_{i}^{+} \\
\lambda_{i}^{-*}
\end{array}\right) \quad i=1,2 .
$$

The second submatrix, in the basis $\left(\tilde{W}^{-}, R_{u}^{-}\right),\left(\tilde{T}^{+}, \tilde{H}_{u}^{+}\right)$of spinors with R-charge equal to minus electric charge, reads

$$
m_{\rho^{-}}=\left(\begin{array}{cc}
-g_{2} v_{T}+M_{W}^{D} & \frac{1}{\sqrt{2}} g_{2} v_{u} \\
-\frac{1}{\sqrt{2}} \Lambda_{u} v_{u} & -\mu_{u}^{\text {eff, }}+
\end{array}\right)
$$


The diagonalization and transformation to mass eigenstates $\eta_{i}^{ \pm}$is performed by $U^{2}$ and $V^{2}$ as

$$
\begin{array}{rlrl}
U^{2, *} m_{\rho^{-}} V^{2, \dagger}=m_{\rho^{-}}^{\text {diag }}, & \tilde{W}^{-}=\sum_{j=1}^{2} U_{j 1}^{2, *} \eta_{j}^{-}, & R_{u}^{-}=\sum_{j=1}^{2} U_{j 2}^{2, *} \eta_{j}^{-} \\
\tilde{T}^{+}=\sum_{j=1}^{2} V_{1 j}^{2, *} \eta_{j}^{+}, & \tilde{H}_{u}^{+}=\sum_{j=1}^{2} V_{2 j}^{2, *} \eta_{j}^{+}
\end{array}
$$

and the other two physical four-component charginos are built as

$$
\rho_{i}^{-}=\left(\begin{array}{c}
\eta_{i}^{-} \\
\eta_{i}^{+*}
\end{array}\right) \quad i=1,2 .
$$

\subsection{Calculation setup and benchmark points}

An analysis of the phases of fields and parameters in the superpotential eq. (2.5), the softbreaking terms, eqs. (2.7) and (2.12), and the tadpole eqs. (2.16) leads to the conclusion that it is possible to choose $M_{B}^{D}, M_{W}^{D}, M_{O}^{D}, \mu_{d}$ and $\mu_{u}$ positive using the free phases of $\hat{S}, \hat{T}, \hat{\mathcal{O}}, \hat{R}_{d}, \hat{R}_{u}$. It then must be allowed for $v_{S}$ and $v_{T}$ to become positive or negative, as required by the tadpole equations. Also the sign of the couplings $\lambda$ and $\Lambda$ is not fixed. $B_{\mu}$ can be chosen to be positive by the usual PQ-symmetry. All other soft masses need to be positive to avoid unwanted charge or color symmetry breaking. In table $2^{1}$ we define benchmark points which represent regions of the MRSSM parameter space with distinct characteristics. It will be shown in the following sections that they are in agreement with experiment and they will serve as starting points for scans of the parameter space. The SM input parameters are [38]

$$
\begin{array}{rlrl}
\hat{\alpha}^{\overline{\mathrm{MS}}}\left(m_{Z}\right) & =127.940^{-1} & \hat{m}_{b}^{\overline{\mathrm{MS}}}\left(\hat{m}_{b}^{\overline{\mathrm{MS}}}\right) & =4.18 \mathrm{GeV} \\
G_{\mu} & =1.1663787 \times 10^{-5} \mathrm{GeV}^{-2} & m_{t} & =173.34 \mathrm{GeV} \\
m_{Z} & =91.1876 \mathrm{GeV} &
\end{array}
$$

For the computational calculations in the MRSSM the Mathematica [39] package SARAH [40-44] v4.3.5 is used. A model file for the MRSSM defined there has been modified to match with our model definitions as specified in section 2.1. With SARAH, an MRSSM version of the spectrum generator SPheno $[45,46]$ has been created. This allows us to calculate the mass spectrum of the model at the full one-loop level. With the recent framework FlexibleSUSY [47] v1.0.2, a second spectrum generator was generated which has similarities with Softsusy $[48,49]$ and which was used for comparison. The correctness of all calculations was checked at great length, and especially details for the calculation of the Higgs and W boson mass will be presented in the following sections 3 and 4 . In section 5 we will then show how it is possible for MRSSM to accommodate both the lightest Higgs boson mass of $125 \mathrm{GeV}$ and the correct $\mathrm{W}$ boson mass and which regions of parameter space are still viable for the model.

\footnotetext{
${ }^{1}$ Parameters are defined following the SPA convention [37].
} 


\begin{tabular}{|c|c|c|c|}
\hline & BMP1 & BMP2 & BMP3 \\
\hline $\tan \beta$ & 3 & 10 & 40 \\
\hline$B_{\mu}$ & $500^{2}$ & $300^{2}$ & $200^{2}$ \\
\hline$\lambda_{d}, \lambda_{u}$ & $1.0,-0.8$ & $1.1,-1.1$ & $0.15,-0.15$ \\
\hline$\Lambda_{d}, \Lambda_{u}$ & $-1.0,-1.2$ & $-1.0,-1.0$ & $-1.0,-1.15$ \\
\hline$M_{B}^{D}$ & 600 & 1000 & 250 \\
\hline$m_{R_{u}}^{2}$ & $2000^{2}$ & $1000^{2}$ & $1000^{2}$ \\
\hline$\mu_{d}, \mu_{u}$ & \multicolumn{3}{|c|}{400,400} \\
\hline$M_{W}^{D}$ & \multicolumn{3}{|c|}{500} \\
\hline$M_{O}^{D}$ & \multicolumn{3}{|c|}{1500} \\
\hline$m_{T}^{2}, m_{S}^{2}, m_{O}^{2}$ & \multicolumn{3}{|c|}{$3000^{2}, 2000^{2}, 1000^{2}$} \\
\hline$m_{Q ; 1,2}^{2}, m_{Q ; 3}^{2}$ & \multicolumn{3}{|c|}{$2500^{2}, 1000^{2}$} \\
\hline$m_{D ; 1,2}^{2}, m_{D ; 3}^{2}$ & \multicolumn{3}{|c|}{$2500^{2}, 1000^{2}$} \\
\hline$m_{U ; 1,2}^{2}, m_{U ; 3}^{2}$ & \multicolumn{3}{|c|}{$2500^{2}, 1000^{2}$} \\
\hline$m_{L}^{2}, m_{E}^{2}$ & \multicolumn{3}{|c|}{$1000^{2}$} \\
\hline$m_{R_{d}}^{2}$ & \multicolumn{3}{|c|}{$700^{2}$} \\
\hline$v_{S}$ & 5.9 & 1.3 & -0.14 \\
\hline$v_{T}$ & -0.33 & -0.19 & -0.34 \\
\hline$m_{H_{d}}^{2}$ & $671^{2}$ & $761^{2}$ & $1158^{2}$ \\
\hline$m_{H_{u}}^{2}$ & $-532^{2}$ & $-544^{2}$ & $-543^{2}$ \\
\hline
\end{tabular}

Table 2. Benchmark points. Dimensionful parameters are given in $\mathrm{GeV}$ or $\mathrm{GeV}^{2}$, as appropriate. The first part gives input parameters, where the values are specific for each point, the second, where the values are common for all points. The last part shows the derived parameters.

\section{Higgs boson mass prediction at one loop}

In section 2 it was noted that mixing with the additional scalars can reduce the tree-level lightest Higgs boson mass significantly, see eq. (2.20). Therefore, loop corrections to the Higgs boson mass play even more significant role than in the MSSM [50-52]. New, nonMSSM loop corrections can balance the tree-level reduction and can be helpful in lowering the fine-tuning in the model. ${ }^{2}$

To calculate the Higgs boson pole mass at the one-loop level, we renormalize the parameters of the model in the $\overline{\mathrm{DR}}$ scheme and choose $v_{d}, v_{u}, v_{S}$ and $v_{T}$ to be given by the minimum of the loop-corrected effective potential. ${ }^{3}$ Then the pole mass $m_{\text {pole }}^{2}$ of a field is given by the pole of the full propagator

$$
0 \stackrel{!}{=} \operatorname{det}\left[p^{2} \delta_{i j}-\hat{m}_{i j}^{2}+\Re\left(\hat{\Sigma}_{i j}\left(p^{2}\right)\right)\right]_{p^{2}=m_{\mathrm{pole}}^{2}},
$$

where $p$ is the momentum, $\hat{m}^{2}$ the tree-level mass matrix and $\hat{\Sigma}\left(p^{2}\right)$ the finite part of

\footnotetext{
${ }^{2}$ For the discussion of fine-tuning in the MRSSM we refer to [34].

${ }^{3}$ This is the definition commonly used and for which the results of refs. [53, 54] apply; for a recent comparison with alternatives, see ref. [55].
} 
the self-energy corrections. Here and in the following sections, mass parameters with hats denote $\overline{\mathrm{DR}}$-renormalized tree-level quantities fulfilling the relations of section 2 , while mass parameters without hats denote loop-corrected pole masses.

\subsection{Full one-loop calculation}

The one-loop self energies have been computed exactly using FeynArts [56], FormCalc $[57,58]$ and Feynman rules generated by SARAH. They are also implemented in the spectrum generators generated by SARAH and by FlexibleSUSY as described in section 2.4. We have verified that all three implementations agree. As a further check we computed the self energies in the effective potential approximation (see below) and found agreement for the gauge-independent part.

Therefore, eq. (3.1) can be analyzed at complete one-loop order. Due to the momentum dependence of self-energies, no direct solution for $m_{\text {pole }}^{2}$ can be given, but has to be found iteratively.

\subsection{Effective potential approach}

It is beneficial to complement the full calculation with a compact approximation of the leading behavior. Loop contributions to the Higgs boson masses can be approximated by neglecting the $p^{2}$ dependence within the self-energy in eq. (3.1). Then the self energy $\hat{\Sigma}(0)$ can be obtained from the second derivatives of the effective potential [59], which is given by

$$
V_{\mathrm{eff}}^{1 \mathrm{~L}}=\frac{1}{64 \pi^{2}} \sum_{i}(-1)^{2 S_{i}+1}\left(2 S_{i}+1\right) \operatorname{Tr}\left[M_{i}^{4}\left(\log \frac{M_{i}^{2}}{Q^{2}}-\frac{3}{2}\right)\right],
$$

summing over all fields with Higgs field dependent mass matrix $M_{i}^{2}$ and spin $S_{i}$ in the $\overline{\mathrm{DR}}$ scheme and Landau gauge. As no implicit dependence on the momentum remains, eq. (3.1) can be solved directly for the one-loop corrected mass matrix:

$$
m_{\text {pole, approx } ; i j}^{2}=\hat{m}_{i j}^{2}+\left.\frac{\partial^{2} V_{\text {eff }}^{1 L}}{\partial \phi_{i} \partial \phi_{j}}\right|_{\phi_{i}=0, \phi_{j}=0}
$$

Eigenvalues of this matrix give then an approximation to the pole masses of corresponding mass eigenstates.

For illustration we use again the limit leading to eq. (2.20), $\lambda=\lambda_{u}=-\lambda_{d}, \Lambda=$ $\Lambda_{u}=\Lambda_{d}$ and $v_{S} \approx v_{T} \approx 0$, and assume large $\tan \beta$. Then, the lightest Higgs state is given mainly by the $\phi_{u}$ component and only the $\left(\phi_{u}, \phi_{u}\right)$-component of the mass matrix in eq. (2.19) needs to be considered. Simple analytical expressions can then be derived by expanding $V_{\text {eff }}^{1 \mathrm{~L}}$ in powers of $\phi_{u}$, where terms of higher order than $\mathcal{O}\left(\phi_{u}^{4}\right)$ will be suppressed by denominators containing $m_{S}^{2}$ and $m_{T}^{2}$. 
The most important contributions are the ones governed by four powers of $\lambda / \Lambda$. The one-loop terms are given by

$$
\begin{aligned}
\Delta m_{H_{1}, \text { eff.pot }, \lambda}^{2}= & \frac{2 v^{2}}{16 \pi^{2}}\left[\frac{\Lambda^{2} \lambda^{2}}{2}+\frac{4 \lambda^{4}+4 \lambda^{2} \Lambda^{2}+5 \Lambda^{4}}{8} \log \frac{m_{R_{u}}^{2}}{Q^{2}}\right. \\
& +\left(\frac{\lambda^{4}}{2}-\frac{\lambda^{2} \Lambda^{2}}{2} \frac{m_{S}^{2}}{m_{T}^{2}-m_{S}^{2}}\right) \log \frac{m_{S}^{2}}{Q^{2}} \\
& +\left(\frac{5}{8} \Lambda^{4}+\frac{\lambda^{2} \Lambda^{2}}{2} \frac{m_{T}^{2}}{m_{T}^{2}-m_{S}^{2}}\right) \log \frac{m_{T}^{2}}{Q^{2}} \\
& -\left(\frac{5}{4} \Lambda^{4}-\lambda^{2} \Lambda^{2} \frac{\left(M_{W}^{D}\right)^{2}}{\left(M_{B}^{D}\right)^{2}-\left(M_{W}^{D}\right)^{2}}\right) \log \frac{\left(M_{W}^{D}\right)^{2}}{Q^{2}} \\
& \left.-\left(\lambda^{4}+\lambda^{2} \Lambda^{2} \frac{\left(M_{B}^{D}\right)^{2}}{\left(M_{B}^{D}\right)^{2}-\left(M_{W}^{D}\right)^{2}}\right) \log \frac{\left(M_{B}^{D}\right)^{2}}{Q^{2}}\right] .
\end{aligned}
$$

This result agrees with ver. 2 of [34]. Note that the logarithmic terms have a similar form as the stop-top contributions

$$
\Delta m_{H_{1}, \text { eff.pot }, y_{t}}^{2}=\frac{6 v^{2}}{16 \pi^{2}}\left[Y_{t}^{4} \log \left(\frac{m_{\tilde{t}_{1}} m_{\tilde{t}_{2}}}{m_{t}^{2}}\right)\right] .
$$

This, of course, can be explained with similarity of the Yukawa and $\lambda / \Lambda$ terms in the superpotential in combination with the $\hat{H}_{u}$ field.

For the numerical analysis no approximation and expansions are performed and the full dependencies of the effective potential are taken into account.

\subsection{Comparison of different calculations and higher-order uncertainties}

In the plots of figure 1 the different predictions (tree-level, effective potential, full one-loop) for the lightest Higgs boson mass are shown as functions of one of the couplings $\Lambda_{u}$ (solid), $\lambda_{u}$ (dashes), and $\Lambda_{d}$ (dots). For every line, the corresponding parameter is varied and all the others are set to values given by benchmark point 1 (left plot) and 3 (right plot) from table 2. ${ }^{4}$ As described in section 2.2, the tree-level mass (magenta) is reduced significantly below $m_{Z} \cos \beta$ for large values of $\Lambda_{u}$ and $\lambda_{u}$ as the mixing of the doublets with the singlet and triplet components gets enhanced. The parameter $\Lambda_{d}$ generally has no strong influence on the prediction even for a low $\tan \beta=3$. The general asymmetry of the plots with regard to the sign of the $\Lambda$ 's originates from the dependence of the off-diagonal elements in the tree-level Higgs boson mass matrix.

The one-loop effective potential (blue) and full one-loop (red) predictions both show the large positive contribution from top Yukawa as well as the $\Lambda / \lambda$ enhancement. As described by eq. (2.20), the tree-level Higgs boson mass will show a quadratic dependence on the $\Lambda / \lambda$, while the one-loop contribution given by eq. (3.4) has a quartic dependence, explaining the behavior of the sum.

\footnotetext{
${ }^{4}$ For large couplings $\lambda / \Lambda$ the mixing with singlet and/or triplet states gets too strong and tachyonic states appear at tree level. The lines in the plots end at those values of $\lambda / \Lambda$ beyond which this happens.
} 

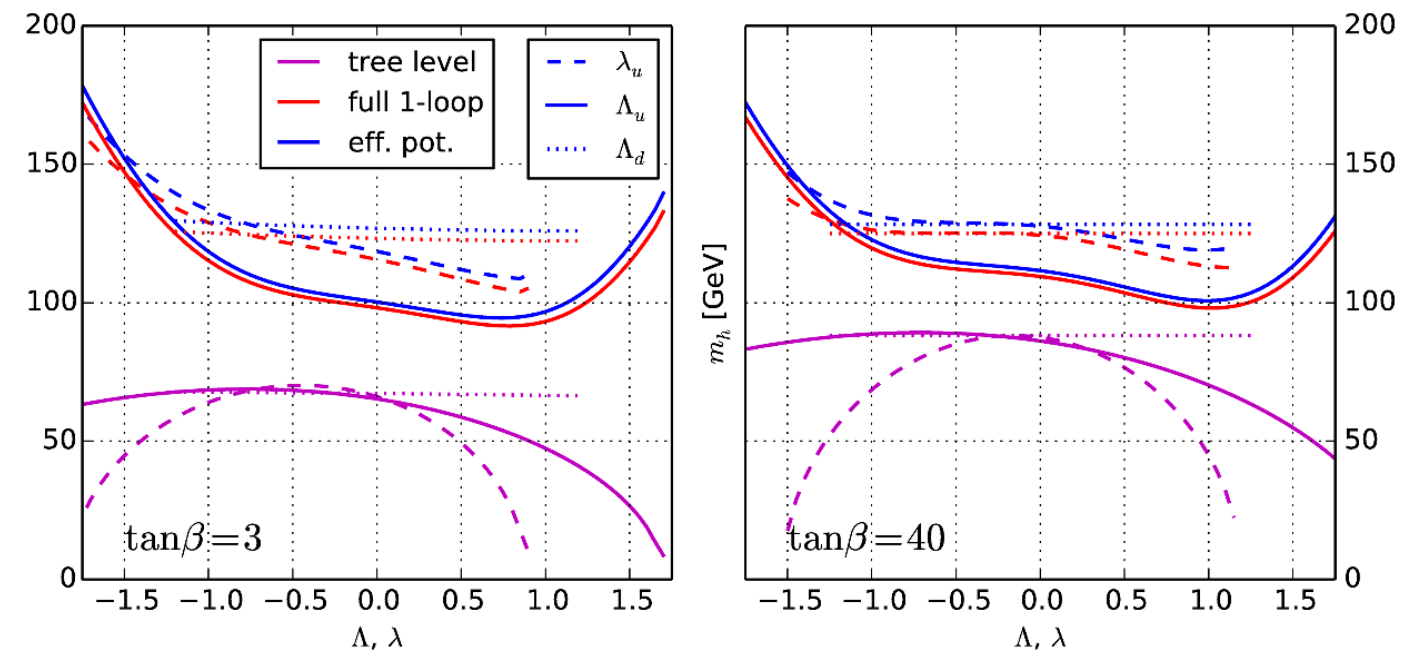

Figure 1. Comparison of the lightest Higgs boson mass calculated using the effective potential approach (blue) and the full one-loop calculation (red), as well as the tree-level mass (magenta). Results are shown as functions of one of the couplings: $\Lambda_{u}$ (solid), $\Lambda_{d}$ (dots), $\lambda_{u}$ (dashes), for benchmark point 1 (left, $\tan \beta=3$ ) and 3 (right, $\tan \beta=40$ ).

The contribution from the top/stop Yukawa alone can be read from the solid lines at $\Lambda_{u}=0$, since then only stop/top contributions are significant. It can be seen that in the MRSSM a stop mass of one $\mathrm{TeV}$, as set in the benchmark points, is not enough to achieve the correct value of the Higgs boson mass, due to the absence of left-right mixing. But the additional $\Lambda / \lambda$ contributions can push it to the experimental result for values of $\Lambda_{u}$ close to unity. ${ }^{5}$ This is clearly seen in table 3 , where contributions from different sectors of the MRSSM model to the lightest Higgs boson mass are shown. In particular, the low values of $\tan \beta$ require a larger value of $\Lambda$ and heavier R-Higgs bosons to compensate the significant reduction of the tree-level value.

To get an estimate of the higher-order corrections, we follow the discussion in [60]. There, two-loop corrections from the strongly interacting sector and their implementation in different spectrum generators for the MSSM and, among other things, different higher order contributions in the $\overline{\mathrm{DR}}$ scheme are presented. These two-loop corrections are usually positive and contribute around $3-5 \mathrm{GeV}$. Similar effects from the strongly interacting sector should also exists in the MRSSM. Calculating in the $\overline{\mathrm{DR}}$ scheme we expect similar positive corrections of order $\mathcal{O}\left(\alpha_{S} \alpha_{t}\right)$. Although quantitative differences due to the Dirac nature of the gluino in the MRSSM might appear, the main contribution from gluon diagrams should be identical. Effects of order $\mathcal{O}\left(\alpha_{t}^{2}\right)$ should behave similar to the MSSM, with small and usually negative contributions, see table 5 of [60].

As in the one-loop calculation, we expect that effects of the order $\mathcal{O}\left(\alpha_{\Lambda}^{2}+\alpha_{\lambda}^{2}+\alpha_{\Lambda} \alpha_{\lambda}\right)$ will have a similar impact as these subleading effects, due to the already noted similarity of

\footnotetext{
${ }^{5}$ Note that we define $\Lambda_{u / d}$ differing by a factor of $\sqrt{2}$ compared to the conventional form of a Yukawa term due to our normalization of the triplet field.
} 


\begin{tabular}{|l|cccccc|}
\hline & gauge & gluino/sgluon & chargino & R-Higgs & all \\
& & $\begin{array}{c}\text { ghost } \\
\text { tree-level }\end{array}$ & $\begin{array}{c}\text { nuark/squark } \\
\text { neutralino }\end{array}$ & & \\
\hline BMP1 & 69.9 & 81.5 & 110.3 & 117.8 & 124.2 & 125.3 \\
BMP2 & 86.5 & 96.3 & 120.7 & 123.2 & 124.1 & 125.1 \\
BMP3 & 90.6 & 93.9 & 118.4 & 123.4 & 124.1 & 125.1 \\
\hline
\end{tabular}

Table 3. The lightest Higgs boson mass (in $\mathrm{GeV}$ ) for the benchmark points: tree-level value and after adding one-loop contributions sector by sector of the MRSSM.

the parameters in the superpotential. A quantitative analysis of all these two-loop effects is left for future work and we estimate a theoretical uncertainty for the lightest Higgs boson mass of $6 \mathrm{GeV}$ for our one-loop mass calculation, based on an expected two-loop contribution of up to $5 \mathrm{GeV}$ and the two-loop uncertainty of $1-3 \mathrm{GeV}$ given in [60].

\section{W boson mass prediction at one loop}

The mass of the $\mathrm{W}$ boson is one of the most precisely known electroweak precision observables and plays a crucial role in constraining extensions of the SM. The SM and MSSM loop corrections to $m_{W}$ are dominated by the top Yukawa coupling. We have seen that the new superpotential couplings $\lambda_{u, d}, \Lambda_{u, d}$ of the MRSSM influence the Higgs boson mass similarly to the top Yukawa coupling - hence it is to be expected that the $\mathrm{W}$ boson mass has a significant dependence on these new couplings. Here we present the higher-order computation of the $\mathrm{W}$ boson mass in the MRSSM, which can then be used to constrain the MRSSM parameter space.

\subsection{Master formula for $m_{W}$}

Like for the Higgs boson mass, an appropriate scheme is the $\overline{\mathrm{DR}}$ scheme. The calculation can then be organized as in ref. [61] for the SM; a major difference is the appearance of the additional Higgs triplet with non-vanishing vev, which enters already at lowest order (for computations of $m_{W}$ in models with Higgs triplets and singlets see refs. [62, 63] and [64], respectively).

Beyond tree-level the $\mathrm{W}$ boson pole mass $m_{W}$ can be obtained from the precisely known muon decay constant using the relation

$$
\frac{G_{\mu}}{\sqrt{2}}=\frac{\pi \hat{\alpha}}{2 \hat{s}_{W}^{2} m_{W}^{2}} \frac{1}{1-\Delta \hat{r}_{W}}
$$

As in the previous section, $\hat{\alpha}, \hat{s}_{W}^{2}=\hat{g}_{1}^{2} /\left(\hat{g}_{1}^{2}+\hat{g}_{2}^{2}\right)$ are $\overline{\mathrm{DR}}$-renormalized running parameters of the MRSSM. The denominator $\left(1-\Delta \hat{r}_{W}\right)$ contains quantum corrections from the W boson self-energy, process dependent box- and vertex-type contributions and counterterms; it also properly resums leading two-loop SM corrections [61]. 
Expressing the weak mixing angle as $\hat{s}_{W}^{2}=1-\frac{m_{W}^{2}}{m_{Z}^{2} \hat{\rho}}$ in terms of the parameter $\hat{\rho}$, defined as

$$
\hat{\rho}=\frac{m_{W}^{2}}{m_{Z}^{2} \hat{c}_{W}^{2}}
$$

we obtain the master formula for the $\mathrm{W}$ boson mass

$$
m_{W}^{2}=\frac{1}{2} m_{Z}^{2} \hat{\rho}\left[1+\sqrt{1-\frac{4 \pi \hat{\alpha}}{\sqrt{2} G_{\mu} m_{Z}^{2} \hat{\rho}\left(1-\Delta \hat{r}_{W}\right)}}\right] .
$$

Hence we need to compute the three quantities $\hat{\alpha}, \hat{\rho}$, and $\Delta \hat{r}_{W}$, all of which depend on the entire particle content of the model. Before discussing these computations it is instructive to expand eq. (4.3) in small shifts by writing each of the quantities $q=m_{W}, \hat{\alpha}, \hat{\rho}, \Delta \hat{r}_{W}$ in the form of $q=q^{\text {ref }}+\delta(q)$. We then obtain

$$
m_{W} \approx m_{W}^{\mathrm{ref}}+\frac{m_{Z} \hat{c}_{W}}{2\left(\hat{c}_{W}^{2}-\hat{s}_{W}^{2}\right)}\left[\hat{c}_{W}^{2} \delta(\hat{\rho})-\hat{s}_{W}^{2}\left(\delta\left(\Delta \hat{r}_{W}\right)+\delta(\hat{\alpha})\right)\right]
$$

which shows the relative importance of these contributions. Note that the form of eq. (4.3) resums leading two-loop corrections, and that there are cancellations between the terms on the right-hand side of eq. (4.4).

\subsection{Computational framework}

We now describe the computation of the three quantities $\hat{\alpha}, \hat{\rho}, \Delta \hat{r}_{W}$. The $\overline{\mathrm{DR}}$ running electromagnetic coupling $\hat{\alpha}$ in the MRSSM can be obtained from the known running SM coupling $\hat{\alpha}^{\overline{\mathrm{MS}}, \mathrm{SM}}\left(m_{Z}\right)$ by applying MRSSM threshold corrections and adding finite counterterm which converts from $\overline{\mathrm{MS}}$ to $\overline{\mathrm{DR}}$. These are

$$
\begin{aligned}
\frac{2 \pi}{\alpha} \Delta \hat{\alpha}^{\overline{\mathrm{DR}}, \operatorname{MRSSM}}\left(m_{Z}\right)= & \frac{1}{3}-\sum_{i=1}^{6}\left(\frac{1}{3} \log \frac{m_{\tilde{l}_{i}^{ \pm}}}{m_{Z}}+\frac{1}{9} \log \frac{m_{\tilde{d}_{i}}}{m_{Z}}+\frac{4}{9} \log \frac{m_{\tilde{u}_{i}}}{m_{Z}}\right) \\
& -\sum_{i=1}^{3}\left(\frac{4}{3} \log \frac{m_{l_{i}^{ \pm}}}{m_{Z}}+\frac{4}{9} \log \frac{m_{d_{i}}}{m_{Z}}+\frac{16}{9} \log \frac{m_{u_{i}}}{m_{Z}}\right) \\
& -\sum_{i=1}^{3} \frac{1}{3} \log \frac{m_{H_{i}^{ \pm}}}{m_{Z}}-\sum_{i=1}^{2} \frac{1}{3} \log \frac{m_{R_{i}^{ \pm}}}{m_{Z}}-\sum_{i=1}^{2} \frac{4}{3}\left(\log \frac{m_{\chi_{i}^{ \pm}}}{m_{Z}}+\log \frac{m_{\rho_{i}^{ \pm}}}{m_{Z}}\right)
\end{aligned}
$$

where $\alpha$ is the electromagnetic coupling in the Thomson limit. In our case this expression is always negative, reducing the value of the running coupling

$$
\hat{\alpha}\left(m_{Z}\right)=\frac{\hat{\alpha}^{\overline{\mathrm{MS}}, \mathrm{SM}}\left(m_{Z}\right)}{1-\Delta \hat{\alpha}^{\hat{\mathrm{DR}}, \operatorname{MRSSM}}\left(m_{Z}\right)} \leq \hat{\alpha}^{\overline{\mathrm{MS}}, \mathrm{SM}}\left(m_{Z}\right)
$$

For the benchmark points defined in section 2.4 we obtain

$$
\hat{\alpha}^{-1}\left(m_{Z}\right) \approx 132 \text {. }
$$

Large corrections to the $\mathrm{W}$ boson mass originate in the $\hat{\rho}$ parameter defined in eq. (4.2). In the SM, the dominant contributions arise from top/bottom loop; in the MRSSM there 
are not only loop contributions but already a tree-level contribution due to the presence of the Higgs triplet with a vev $v_{T}$ as already pointed out in eq. (2.17). This is used to define the tree-level shift $\Delta \hat{\rho}_{\text {tree }}$ using

$$
\hat{\rho}_{\text {tree }}=\frac{\hat{m}_{W}^{2}}{\hat{m}_{Z}^{2} \hat{c}_{W}^{2}} \equiv 1+\Delta \hat{\rho}_{\text {tree }}=1+\frac{4 v_{T}^{2}}{v^{2}} .
$$

Here $\hat{m}_{V}(V=W, Z)$ are the tree-level $\overline{\mathrm{DR}}$ masses given by eq. (2.17) and related to the pole masses $m_{V}$ by

$$
\hat{m}_{V}^{2}=m_{V}^{2}+\Re\left(\hat{\Pi}_{V V}^{T}\left(m_{V}^{2}\right)\right),
$$

where $\hat{\Pi}_{V V}^{T}$ denotes the finite part of the respective transverse vector boson self energy. The loop contributions to $\hat{\rho}$ are given by

$$
\frac{\hat{\rho}}{\hat{\rho}_{\text {tree }}} \equiv \frac{1}{1-\Delta \hat{\rho}}=\frac{1+\frac{\Re\left(\Pi_{Z Z}^{T}\left(m_{Z}^{2}\right)\right)}{m_{Z}^{2}}}{1+\frac{\Re\left(\Pi_{W W}^{T}\left(m_{W}^{2}\right)\right)}{m_{W}^{2}}},
$$

and the full $\hat{\rho}$ can then be approximated by

$$
\hat{\rho}=\frac{1}{1-\Delta \hat{\rho}_{\text {tree }}-\Delta \hat{\rho}},
$$

neglecting products of the form $\Delta \hat{\rho}_{\text {tree }} \Delta \hat{\rho}$.

The remaining quantity $\Delta \hat{r}_{W}$ can then be written as [61]

$$
\begin{aligned}
\Delta \hat{r}_{W} & =\Delta \hat{\rho}(1-\Delta \hat{r})+\Delta \hat{r}, \\
\Delta \hat{r} & =\Re\left(\frac{1}{1-\Delta \hat{\rho}} \frac{\hat{\Pi}_{W W}^{T}(0)}{m_{W}^{2}}-\frac{\hat{\Pi}_{Z Z}^{T}\left(m_{Z}^{2}\right)}{m_{Z}^{2}}\right)+\frac{1}{1-\Delta \hat{\rho}} \delta_{V B} .
\end{aligned}
$$

The term $\delta_{V B}$ contains vertex and box diagram contributions to muon decay; it is detailed in the appendix B. It is worth noting that in these equations only the loop contributions to $\hat{\rho}$ appear. This way of writing the contributions and the master formula (4.3) automatically resums leading reducible two-loop contributions; further leading irreducible SM-like two-loop contributions can also be incorporated easily [61, 65, 66]. This calculation has been implemented numerically with the help of SARAH, appropriately modified to take into account the Higgs triplet contributions.

\subsection{Qualitative discussion}

In our numerical analysis and plots of the $\mathrm{W}$ boson mass we use the full MRSSM one-loop results for $\Delta \hat{\alpha}^{\overline{\mathrm{DR}}, \mathrm{MRSSM}}, \Delta \hat{\rho}, \Delta \hat{r}_{W}$ including leading two-loop contributions as incorporated in eq. (4.1).

Here we discuss the leading behavior, particularly focusing on the dependence on the new parameters $\lambda_{u, d}, \Lambda_{u, d}$. These enter in the sectors involving charginos/neutralinos, Higgs bosons and R-Higgs bosons. The pure one-loop approximation, eq. (4.4), can be recast in a form using the electroweak precision parameters $S, T$ and $U$ [67-72] as

$$
m_{W}=m_{W}^{\mathrm{ref}}+\frac{\hat{\alpha} m_{Z} \hat{c}_{W}}{2\left(\hat{c}_{W}^{2}-\hat{s}_{W}^{2}\right)}\left(-\frac{S}{2}+\hat{c}_{W}^{2} T+\frac{\hat{c}_{W}^{2}-\hat{s}_{W}^{2}}{4 \hat{s}_{W}^{2}} U\right) .
$$


The main contribution to $m_{W}$ from the MRSSM can be described using the $T$ parameter

$$
\hat{\alpha} T=\left.\left(\frac{\hat{\Pi}_{Z Z}^{T}(0)}{\hat{m}_{Z}^{2}}-\frac{\hat{\Pi}_{W W}^{T}(0)}{\hat{m}_{W}^{2}}\right)\right|_{\text {New Physics }}
$$

for realistic scenarios, i.e. for each sector the contribution to the bracket on the r.h.s. of eq. (4.4) is well approximated by $\hat{\alpha} \hat{c}_{W}^{2} T$.

Calculating $S, T$ and $U$ has the additional benefit that theses parameters can be used in the calculation of many electroweak precision observables. Therefore, strong bounds exist from fits to a large number of such observables [38].

In order to qualitatively understand the leading behavior and to further illustrate the complexity and richness of the MRSSM, we will consider all three sectors in turn and derive various limits of phenomenological and conceptual interest. All limits assume large $\tan \beta$ and neglect $v_{d, S, T}$.

- Charginos/neutralinos, interplay of $\Lambda_{u}$ and Dirac masses: in the parameter regions considered in the present paper, the chargino/neutralino sector provides the largest contributions to $m_{W}$, and the most influential parameters are $\Lambda_{u}, \lambda_{u}$ and the Diractype mass parameters $\mu_{u}, M_{W, B}^{D}$. For the simplified case $\lambda_{u}=g_{1}=0$ and $\mu_{u}=M_{W}^{D}$ we obtain

$$
T=\frac{1}{16 \pi \hat{s}_{W}^{2} \hat{m}_{W}^{2}} \frac{v_{u}^{4}}{\left(M_{W}^{D}\right)^{2}}\left[\frac{13 g_{2}^{4}+2 g_{2}^{3} \Lambda_{u}+18 g_{2}^{2} \Lambda_{u}^{2}+2 g_{2} \Lambda_{u}^{3}+13 \Lambda_{u}^{4}}{96}\right]+\mathcal{O}\left(\frac{v_{u}^{4}}{\left(M_{W}^{D}\right)^{4}}\right),
$$

while for the similar case $\Lambda_{u}=g_{2}=0$ and $\mu_{u}=M_{B}^{D}$ we obtain the same formula with an additional prefactor $1 / 5$ and the replacement $\Lambda_{u} \rightarrow \sqrt{2} \lambda_{u}, M_{W}^{D} \rightarrow M_{B}^{D}$. Hence the main dependence on the new superpotential couplings $\Lambda_{u}, \lambda_{u}$ is the one of a fourth-order polynomial.

- Charginos/neutralinos as vector-like fermions: the dependence on the Dirac-type mass parameters can also be understood in a different way. If all couplings $g_{1,2}$, $\lambda_{u, d}, \Lambda_{u, d}$ vanish, the charginos and neutralinos simply correspond to new, vectorlike fermions (a singlet, a triplet, and two doublets) with vanishing $T$. As an example with non-vanishing couplings and mixing we consider the $N=2$ SUSY limit $g_{1}=$ $\sqrt{2} \lambda_{u}=\sqrt{2} \lambda_{d}$ and $g_{2}=\Lambda_{u}=-\Lambda_{d}$. For the vector-like limit we then set $\Lambda_{u}=0$ in the neutralino/chargino tree-level mass matricies and the $\tilde{S}-\tilde{B}$ singlet and the $\tilde{R}_{u^{-}}$ $\tilde{H}_{u}$ doublet mix just like the vector-like fermions considered in ref. [73]. Keeping the masses $\mu_{u}$ and $M_{B}^{D}$ independent, with $x=\mu_{u} / M_{B}^{D}$, we obtain

$$
T=\frac{1}{16 \pi \hat{s}_{W}^{2} \hat{m}_{W}^{2}} \frac{v_{u}^{4} \lambda_{u}^{4}}{\left(M_{B}^{D}\right)^{2}}\left[\frac{\left(x-1-4 x^{2}\right) \log x^{2}}{(1-x)^{5}(1+x)^{2}}-\frac{13-x+17 x^{2}-5 x^{3}}{6(1-x)^{4}(1+x)}\right]+\mathcal{O}\left(\frac{v_{u}^{4}}{\left(M_{B}^{D}\right)^{4}}\right) .
$$

Compared to eq. (4.16), the couplings are fixed but the Dirac-type masses enter individually. In the limit $\mu_{u} \rightarrow M_{B}^{D}$, or $x \rightarrow 1$, this equation reduces to

$$
T=\frac{1}{16 \pi \hat{s}_{W}^{2} \hat{m}_{W}^{2}} \frac{2 v_{u}^{4} \lambda_{u}^{4}}{5\left(M_{B}^{D}\right)^{2}}+\mathcal{O}\left(\frac{v_{u}^{4}}{\left(M_{B}^{D}\right)^{4}}\right)
$$

which can also be obtained from eq. (4.16), applied in the appropriate limit. 
- Charginos/neutralinos, no Dirac masses: the above formulas apply for sufficiently heavy Dirac masses. The opposite case is $M_{B, W}^{D}=\mu_{u, d}=0$. In this limit we obtain

$$
T=\frac{v_{u}^{2}}{16 \pi \hat{s}_{W}^{2} \hat{m}_{W}^{2}}\left[\frac{2 \lambda_{u}^{2}-5 \Lambda_{u}^{2}}{4}+\frac{\Lambda_{u}^{2}\left(2 \lambda_{u}^{2}+3 \Lambda_{u}^{2}\right)}{2 \lambda_{u}^{2}-\Lambda_{u}^{2}} \log \frac{2 \lambda_{u}^{2}+\Lambda_{u}^{2}}{2 \Lambda_{u}^{2}}+" g_{1,2}\right. \text {-terms"]. }
$$

The generic behavior is $\propto \lambda^{2} v^{2}$ as opposed to $\propto \lambda^{4} v^{4} / M_{D}^{2}$ as in the previous formulas. The first term (corresponding to $\Lambda_{u} \rightarrow 0$ ) is identical to the SM top/bottom contribution up to the color factor and replacements $Y_{t} \rightarrow \lambda_{u}, Y_{b} \rightarrow 0$, because of the parallel structure of the $\lambda_{u}$ and $Y_{t}$-terms in the superpotential. The gauge-coupling terms in eq. (4.19) are obtained by the replacements $\sqrt{2} \lambda_{u} \rightarrow g_{1}, \Lambda_{u} \rightarrow g_{2}$, reflecting the structure of the chargino/neutralino mass matrices and the $N=2$ SUSY limit.

- Higgs sector: the Higgs sector contributions depend on the same coupling constants, but the Higgs boson masses are also governed by the soft parameters $m_{S, T}^{2}$, which are large in all our examples. The approximation when using the gauge-less limit and setting $\mu_{u}=\mu_{d}=M_{B}^{D}=M_{W}^{D}=\lambda_{u}=0$ to fulfill the tree-level tadpole equations is given as

$$
T=\frac{v_{u}^{2}}{16 \pi \hat{s}_{W}^{2} \hat{m}_{W}^{2}} \frac{\Lambda_{u}^{4} v_{u}^{2}}{12 m_{T}^{2}}+\mathcal{O}\left(\frac{v_{u}^{4}}{m_{T}^{4}}\right) .
$$

It has a qualitatively similar coupling dependence, but the contribution is significantly smaller than the ones of the chargino/neutralino sector as $m_{T}$ is much greater than $M_{D}^{W}$.

- R-Higgs sector: the contributions from the R-Higgs sector have a structure similar to the stop-sbottom contribution [74],

$$
\begin{aligned}
T \approx & \frac{1}{16 \pi \hat{s}_{W}^{2} \hat{m}_{W}^{2}}\left\{-\sin ^{2} 2 \theta_{R} F_{0}\left(m_{R_{1}}^{2}, m_{R_{2}}^{2}\right)+\cos ^{2} \theta_{R}\left[F_{0}\left(m_{R_{1}}^{2}, m_{R_{1}^{+}}^{2}\right)+(1 \rightarrow 2)\right]\right. \\
& \left.+\sin ^{2} \theta_{R}\left[F_{0}\left(m_{R_{1}}^{2}, m_{R_{2}^{+}}^{2}\right)+(1 \leftrightarrow 2)\right]\right\}
\end{aligned}
$$

where

$$
F_{0}(x, y)=x+y+\frac{2 x y}{x-y} \log \frac{y}{x}
$$

and we have neglected contributions proportional to $\left(v_{T} / v\right)^{2}$. This means that in our case, for large soft masses $m_{R_{u}}^{2}$ and $m_{R_{d}}^{2}$, mixing between the R-Higgs bosons and the mass-splitting between neutral and charged R-Higges is suppressed leading to negligible contributions to $T$.

\subsection{Comparison of full and approximate results}

The previous approximations, particularly eqs. (4.16), (4.21), provide useful qualitative insight into the parameter dependence of $m_{W}$. Figure 2 shows how well various approximations agree quantitatively with the full calculation of $m_{W}$. All lines plotted in the figure contain the full SM contribution, but the MRSSM contributions are taken into account 

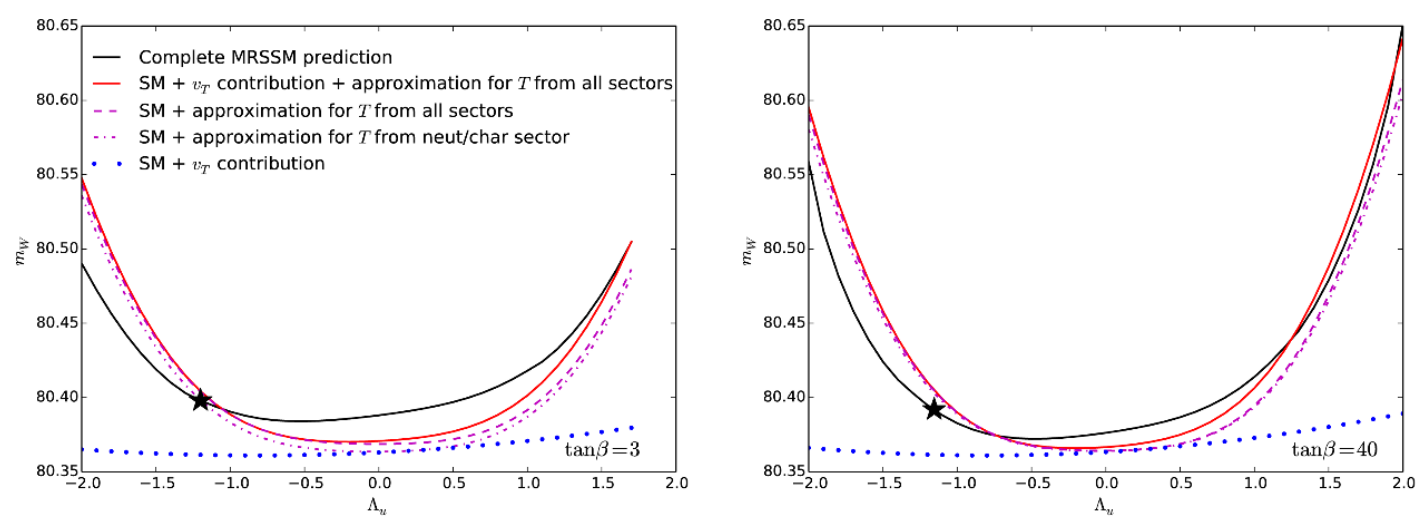

Figure 2. Comparison of the mass of the $\mathrm{W}$ boson depending on $\Lambda_{u}$, calculated using full MRSSM contributions and different approximations for the $T$-parameter (neutralino and chargino sector (neut/char) eq. (4.16), Higgs sector eq. (4.20) and R-Higgs sector eq. (4.21)) and the tree-level contribution from the triplet vev for Benchmark point 1 (left, $\tan \beta=3$ ) and 3 (right, $\tan \beta=40$ ). The black stars mark the corresponding Benchmark points.

\begin{tabular}{|c|ccccc|cccc|cc|c|}
\hline & $H_{2}$ & $A_{1}$ & $H_{1}^{ \pm}$ & $R_{1}$ & $R_{1}^{ \pm}$ & $\chi_{1}$ & $\chi_{2}$ & $\chi_{1}^{ \pm}$ & $\rho_{1}^{ \pm}$ & $\tilde{t}_{1}$ & $\tilde{b}_{1}$ & $\tilde{\nu}$ \\
\hline BMP1 & 897 & 896 & 899 & 912 & 906 & 415 & 420 & 416 & 427 & 1059 & 1061 & 1002 \\
BMP2 & 937 & 937 & 940 & 926 & 921 & 413 & 423 & 413 & 429 & 1061 & 1062 & 1003 \\
BMP3 & 1245 & 1245 & 1248 & 896 & 891 & 251 & 408 & 408 & 424 & 1060 & 1056 & 1000 \\
\hline
\end{tabular}

Table 4. Masses of selected particles (in GeV).

either completely, or only via the $T$-parameter in various approximations, or from the tree-level triplet vev contribution.

We see that the chargino/neutralino approximation (4.16) already gives an excellent approximation to the full $T$-parameter. The $T$-parameter, together with the tree-level triplet vev contribution, provides a good approximation to the full result. The remaining difference from non- $T$-parameter oblique corrections, vertex and box contributions, and leading higher loop contributions, is between $\pm 20 \mathrm{MeV}$, except for $\Lambda_{u} \dot{\iota} 1.5$.

\section{$5 \quad$ Numerical predictions}

In the following we present a thorough analysis of the viable parameter space of the MRSSM. As was shown in sections 3 and 4, the benchmark points of table 2 can accommodate both the mass of the lightest Higgs boson as well as are in agreement with the measured $\mathrm{W}$ boson mass. In the following we will present the mass spectra for the benchmark points and explore the parameter space around them.

\subsection{Mass spectra}

After fixing the critical parameters to account for the Higgs and $W$ boson masses, other parameters of BMP1-BMP3 have been chosen to produce sufficiently heavy states as to not 


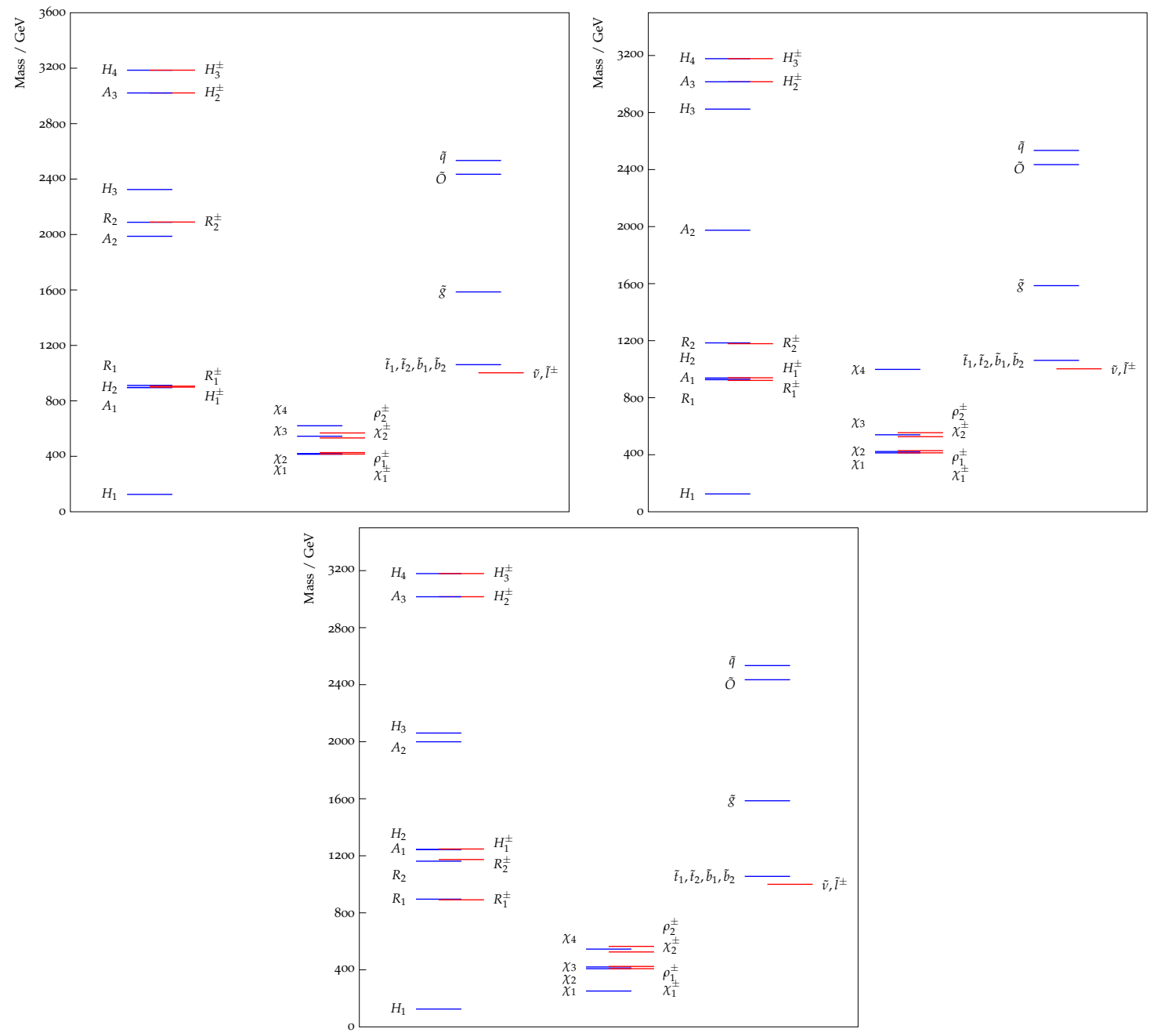

Figure 3. Particle mass spectra for $\tan \beta=3$ (top-left), $\tan \beta=10$ (top-right) and $\tan \beta=40$ (bottom). $\tilde{q}$ denotes all 1st and 2nd generation squarks. Plots done using PySLHA [75].

come into conflict with exclusion bounds from direct collider searches. Figure 3 shows mass spectra for the benchmark points of table 2 and table 4 gives numerical values for lightest states in each sector. The calculation of particle spectra have been performed including one-loop corrections using the SPheno code generated by SARAH. The results have been checked against the ones from FlexibleSUSY, and an agreement of the order of a few percent has been achieved.

A number of comments is in order:

- Heavy charged and neutral $\mathcal{C} \mathcal{P}$-even and odd Higgs bosons are composed mainly from $S$ and $T$ fields. Their masses are driven by large $m_{S}$ and $m_{T}$ soft masses, which in turn are needed to make $v_{S}$ and $v_{T}$ small. ${ }^{6}$ This leads to the hierarchy between them visible in the first column of every plot in figure 3.

\footnotetext{
${ }^{6}$ The mass parameter $m_{S}$ could in principle be much smaller than $m_{T}$ as $v_{S}$ is not as constrained as $v_{T}$.
} 
- Due to the lack of left-right sfermion mixing and choice of common value for soft masses the small mass splitting between left- and right-chiral sfermions is caused by the corresponding fermion masses and the Dirac mass parameters that enter via D-tems, see eq. (2.7). In all BMPs the third-generation squarks and all sleptons are roughly mass degenerate with masses of order $1 \mathrm{TeV}$

- The $R_{2}, R_{2}^{ \pm}$states for the BMP1 are significantly heavier than in BMP2 and BMP3 since the loop-corrections from $R$-Higgs bosons have to account for a lower tree-level mass of the lightest Higgs boson, cf. table 3 .

- In all benchmark points charginos and neutralinos are well below $1 \mathrm{TeV}$; in the BMP3 the lightest neutralino is as light as $\sim 250 \mathrm{GeV}$.

- table 4 shows that many of the new states should be accessible kinematically at the LHC. Note that the Dirac nature of gauginos, together with R-charge conservation, implies distinctly different signatures in comparison to the MSSM. The study of phenomenological implications of our BMPs for the LHC physics is left for future work.

\subsection{Exploring the parameter space}

It is interesting to explore the parameter space around our benchmark points to show the range of parameters satisfying both the Higgs boson mass and $m_{W}$ constraints. Figures 4 and 5 show scans as functions of two chosen parameters with other parameters fixed and the corresponding benchmark point is marked in each plot. The plots are ordered from left to right by benchmark points with rising $\tan \beta$. The green (yellow) band shows the region of parameter space, where the mass of the lightest Higgs boson is $m_{H_{1}}=126 \pm 2( \pm 8) \mathrm{GeV}$. The red contour lines give the mass of the $\mathrm{W}$ boson. The white regions denote regions in which the mass spectra contain tachyonic states.

We first recall that for both the Higgs and $\mathrm{W}$ boson masses $m_{H_{1}}$ and $m_{W}$, large scalar masses (compared to chargino/neutralino masses) are favorable, and the benchmark points are chosen accordingly. The large mass ratio enhances the loop contribution to $m_{H_{1}}$ and suppresses the contribution of the new scalars to $m_{W}$, see the approximations in eqs. (3.4) and (4.21) and comments there. The exact values of the scalar masses are thus not very important, and therefore in all plots they will always remain fixed to their benchmark values.

The plots can be understood by noting that the new contributions to the $m_{W}$ prediction are dominated by the neutralino/chargino contributions to $T$. The parameter dependence of the lightest Higgs boson mass $m_{H_{1}}$ is described by the tree-level reduction, eq. (2.20) and loop corrections, eq. (3.4).

The top row in figure 4 gives the behavior of the parameter combination $\lambda_{u}, \lambda_{d}$. For this combination the change in $\tan \beta$ with the benchmark points has the strongest influence. As is to be expected, the down-type parameter $\lambda_{d}$ has a particularly noteworthy effect for small $\tan \beta$. Nevertheless, even for $\tan \beta=3$, the dependence of both $m_{H_{1}}$ and $m_{W}$ on the 

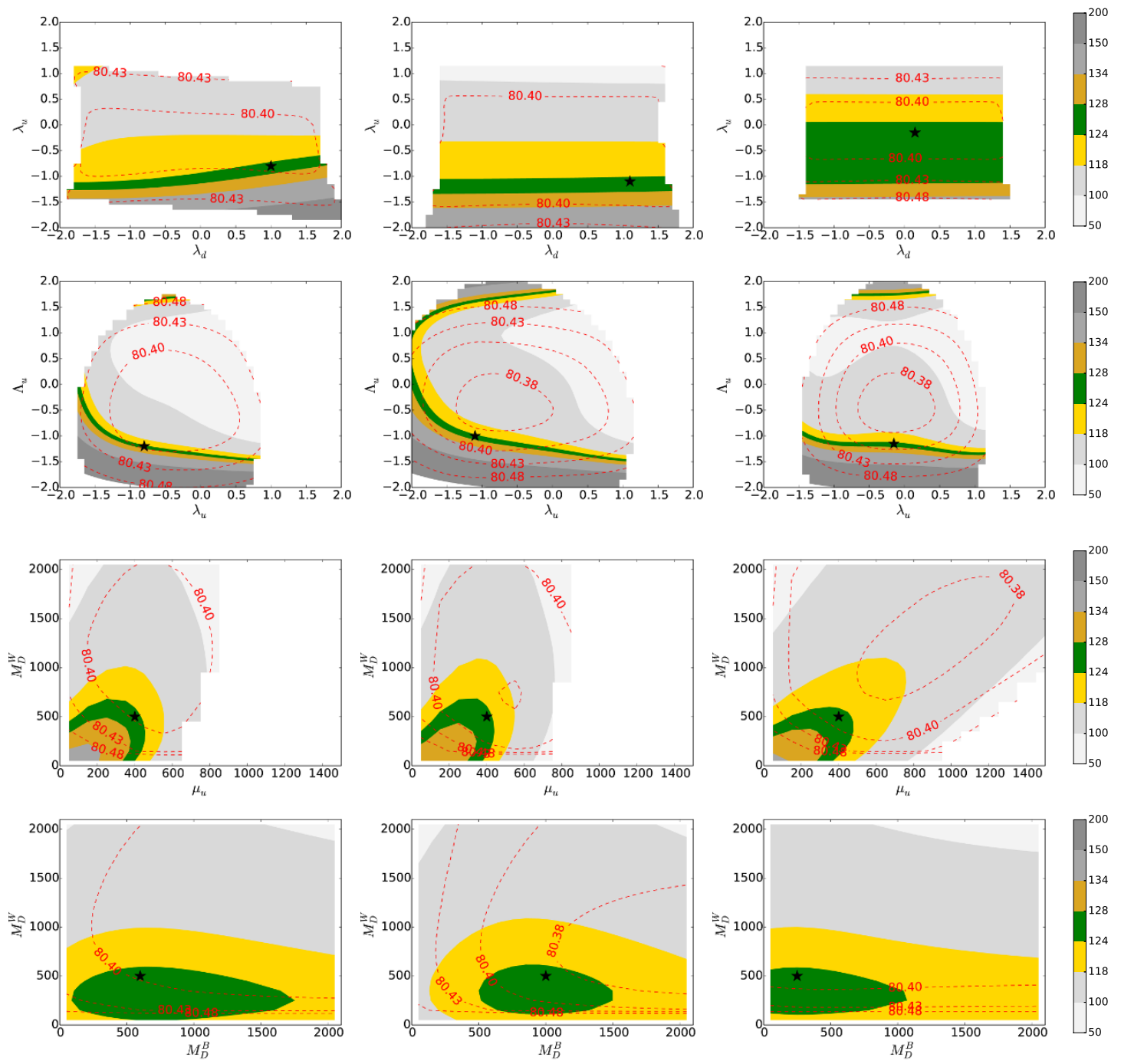

Figure 4. Contour plots showing the behavior of $m_{H_{1}}$ given by the color map and $m_{W}$ given by the red contour lines. The plots are ordered horizontally by benchmark points with rising $\tan \beta$, while vertically different combination of model parameters are varied. The values for the benchmark point of each plot are marked by a star.

up-type parameter $\lambda_{u}$ is much stronger. Therefore, in all the following plots the value of $\lambda_{d}$ is kept fixed to the values of the benchmark points.

The second row in figure 4 shows the $\Lambda_{u}^{4}$ and $\lambda_{u}^{4}$ behavior for $m_{W}$, as expected from the approximate result in eq. (4.16). The Higgs boson mass dependence is also compatible with the previous estimates and figure 1, although some contours in the plots are cut off due to tachyonic states. Both the $\Lambda^{4}, \lambda^{4}$-behavior from eq. (3.4) and the asymmetry in $\Lambda_{u}$ and $\lambda_{u}$ from the tree-level reduction in eq. (2.20) are clearly visible. Owing to the sensitivity of both observables $m_{W, H_{1}}$ to both parameters, the region where both experimental constraints are satisfied is quite narrow, but agreement with experiment is possible. 

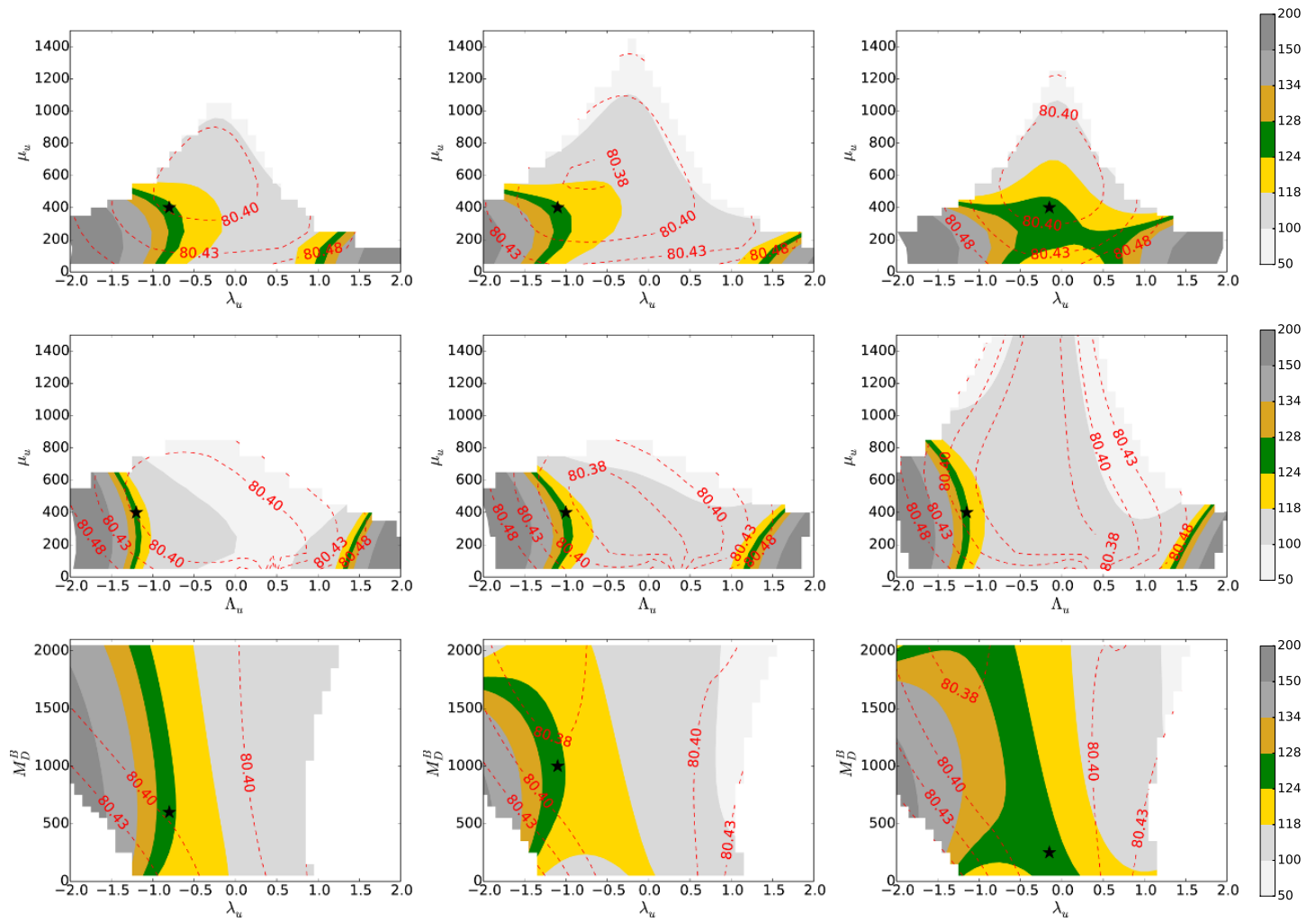

Figure 5. Contour plots with labeling and ordering as for figure 4.

In the third row the scan over the two Dirac mass parameters $\mu_{u}$ and $M_{D}^{W}$ is shown. The correct Higgs boson mass can be found in a region where the tree-level reduction, eq. (2.20), is sufficiently small. The tree-level reduction stays small for sufficiently small Dirac masses (compared to the scalar masses $m_{T, S}$ ), resulting in an elliptic shape of the contours of correct Higgs boson mass (the axes are controlled by the ratio of $g_{2}$ and $\Lambda_{u}$ ). The behaviour of $m_{W}$ can be described using the vector-like limit in eq. (4.17). The Higgs boson mass prefers smaller values of the Dirac masses, while the $\mathrm{W}$ boson mass prefers larger values, but there is a significant overlap.

The lower row in figure 4 shows the behavior with respect the two Dirac gaugino masses $M_{D}^{B}, M_{D}^{W}$. As in the previous plots, too large Dirac masses reduce the loop contributions to $m_{H_{1}}$ significantly and contribute strongly to the tree-level reduction, so that it is not possible to reach the correct Higgs boson mass. This is the main driving force for relatively light gauginos in all benchmark points. Note however that too small Dirac masses provide too large contributions to $m_{W}$, as can be understood from the $T$-parameter approximation in the vector-like limit. It should be added that the impact of the bino mass parameter $M_{D}^{B}$ is far less important than the one of the wino mass parameter $M_{D}^{W}$.

Figure 5 shows the parameter planes $\left(\mu_{u}, \lambda_{u}\right),\left(\mu_{u}, \Lambda_{u}\right)$, and $\left(M_{D}^{B}, \lambda_{u}\right)$. It confirms the fact that the $\lambda / \Lambda$ couplings are most important. The $\Lambda_{u}^{4}$ and $\lambda_{u}^{4}$ behavior is again visible; the $\tan \beta=40$ plot in the top row shows a rather flat behavior of $m_{H_{1}}$ for a range of $\lambda_{u}$, which is equivalent to the $\lambda_{u}$-dependence in figure 1 . 


\begin{tabular}{|l|ccc|}
\hline & BMP1 & BMP2 & BMP3 \\
\hline$m_{H_{1}}$ & $125.3 \mathrm{GeV}$ & $125.1 \mathrm{GeV}$ & $125.1 \mathrm{GeV}$ \\
$m_{W}$ & $80.399 \mathrm{GeV}$ & $80.385 \mathrm{GeV}$ & $80.393 \mathrm{GeV}$ \\
HiggsBounds's obsratio & 0.61 & 0.61 & 0.63 \\
HiggsSignals's p-value & 0.42 & 0.40 & 0.40 \\
$S$ & 0.0097 & 0.0092 & 0.0032 \\
$T$ & 0.090 & 0.091 & 0.085 \\
$U$ & 0.00067 & 0.00065 & 0.0010 \\
Vevacious & $\checkmark$ & $\checkmark$ & $\checkmark$ \\
selected $b$ physics observables & $\checkmark$ & $\checkmark$ & $\checkmark$ \\
\hline
\end{tabular}

Table 5. Collection of different predictions for the benchmark points defined in 2. For details see the full text.

Generally, the plots in figure 5 explicitly show that changes in the Dirac masses can be compensated by changes in $\lambda_{u} / \Lambda_{u}$ such that $m_{W, H_{1}}$ agree with experiment. The required values of $\lambda_{u}$ or $\Lambda_{u}$ are typically close to -1 ; their absolute values are thus similar to the top Yukawa coupling. In particular, the plots again show that the higgsino Dirac mass $\mu_{u}$ has larger influence than the bino one, $M_{D}^{B}$. In the first two rows of figure 5 one of the couplings $\Lambda_{u} / \lambda_{u}$ is varied against $\mu_{u}$. The dependence of $m_{H_{1}}$ on $\mu_{u}$ shows a structure which again can be understood by the reduction formula, eq. (2.20), while the vector-like approximation of $T$ (4.17) for $m_{W}$. Here, the minimum of $T$ is given by $\mu_{u}=M_{D}^{W}$ and the contributions are increasing for a larger mass splitting. The last row of figure 5 shows the variation of $\lambda_{u}$ and $M_{D}^{B}$ and makes it clear that the bino Dirac mass only has a mild influence on the mass predictions, also justifying that the minimum of $T$ as a function of $\mu_{u}$ in the plots before is given by $M_{D}^{W}$ instead.

All these plots show that a suitable range for the input parameters exists to meet the correct value for $m_{W}$ and $m_{H_{1}}$ but that it is non-trivial to find regions where both constraints are fulfilled. These regions are characterized by at least one large $\lambda / \Lambda$ coupling, high scalar masses to reduce the tree-level reduction of $m_{H_{1}}$ and minimize the $T$ parameter, as well as relatively low higgsino and gaugino Dirac masses to minimize tree-level reduction.

\subsection{Experimental constraints}

Table 5 shows a compilation of different predictions for our benchmark points. As argued in the introduction, the MRSSM can accommodate proper Higgs boson mass of around $125 \mathrm{GeV}$ with relatively light stops of around $1 \mathrm{TeV}$, without any left-right mixing which is absent in the MRSSM. The $\mathrm{W}$ boson mass is also found in agreement with the experimental value from combined LEP and Tevatron measurements $m_{W}^{\exp }=80.385 \pm 0.015 \mathrm{GeV}[38]$. This can be compared with the SM theory prediction $m_{W}^{\text {theory,SM }}=80.362 \pm 0.010 \mathrm{GeV}[76,77]$. The Higgs sector of the benchmark points was checked against existing experimental data using HiggsBounds [78-81] v4.1.3 and HiggsSignals $[82,83]$ v1.2.0. The corresponding results for the statistical significance of exclusion are given in table 5 and show that all benchmark points are not excluded by the 


\begin{tabular}{|c|ccccccc|}
\hline & $\gamma \gamma$ & $\operatorname{gg}$ & $W^{ \pm} W^{\mp *}$ & $Z Z^{*}$ & $\tau^{+} \tau^{-}$ & $c \bar{c}$ & $b \bar{b}$ \\
\hline BMP1 & 0.909 & 1.003 & 0.999 & 0.999 & 1.212 & 0.980 & 1.212 \\
BMP2 & 0.917 & 1.023 & 0.999 & 0.999 & 1.192 & 0.998 & 1.192 \\
BMP3 & 0.906 & 1.027 & 1 & 1 & 1.130 & 1 & 1.130 \\
\hline
\end{tabular}

Table 6. Normalized (squared) effective couplings of the lightest Higgs particle to gauge boson and fermion pairs at leading order.

experimental data included in those codes.

In section 4 we described that the main contribution to $m_{W}$ from the MRSSM can be effectively described using the $S, T, U$ parameters. The values of the three parameters for our BMPs given in table 5 fulfill the expectation that the $T$ parameters gives the largest contribution in eq. (4.14), while the next-to-largest is always given by $S$ and an order of magnitude smaller. All values are allowed by the fits to electroweak precision observables.

The Higgs potential of the MRSSM was checked for possible presence of deeper minima in the $\left\{v_{d}, v_{u}, v_{S}, v_{T}\right\}$ space using Vevacious [84] v1.1.00. Within the validity of approximations used by Vevacious, none were found. Searches for deeper charge- and/or color-breaking minima were not performed, as we expect none of them due to the absence of A-terms in the MRSSM [85].

We checked also selected low energy $b$-physics observables, namely $B \rightarrow X_{s} \gamma$, $B_{s / d} \rightarrow \mu^{+} \mu^{-}$and $\Delta M_{B_{s / d}}$ using SARAH's FlavorKit interface and found agreement with measurements, as expected from [14]. Finally the effective couplings of the lightest Higgs boson to quarks, leptons and gauge bosons, normalized to the SM value, are listed in table 6 . The enhanced couplings to the down-type fermions result from the admixture of $\phi_{d}$ to the lightest Higgs boson.

\section{Conclusions}

Models with a continuous R-symmetry contain the complete set of possible types of symmetries in a relativistic quantum field theory. They are phenomenologically appealing for a variety of reasons, e.g. they solve the SUSY flavor problem and relax direct search limits from the LHC. The minimal model, the MRSSM, contains many new states, in particular R-Higgs doublet and singlet/triplet superfields, whose fermionic components partner with the usual higgsinos and $\mathrm{SU}(2) \times \mathrm{U}(1)$ gauginos to give Dirac-type gaugino and higgsino masses.

In this paper we have shown how the MRSSM can accommodate the measured values of the Higgs boson and W boson masses. We have computed both observables via the full one-loop calculations of Higgs boson self energies and muon decay. The most important new model parameters are the superpotential couplings $\lambda_{u, d}, \Lambda_{u, d}$. They enter in a way similar to the top/bottom Yukawa couplings, with the role of the quark doublets and singlets played by the R-Higgs doublets and the singlet/triplet.

For the case of the Higgs boson mass, the $\lambda / \Lambda$-contributions have essentially the same structure as the top/stop contribution. If the $\lambda / \Lambda$ 's are of the order of the top Yukawa 
couplings, the Higgs boson mass can easily be as large as $125 \mathrm{GeV}$ or even larger for a broad range of $\tan \beta$. Stop masses beyond the $\mathrm{TeV}$ are not required.

For the case of the $\mathrm{W}$ boson mass, there exists a limit (for vanishing Dirac higgsino/gaugino masses) in which the $\lambda / \Lambda$-contributions have the same structure as the ones from the top/bottom sector. However, in the more likely case of non-negligible Dirac mass parameters (which have no counterpart in the quark sector), the behavior is different. The interplay between the $\lambda / \Lambda$ 's and the Dirac mass parameters has a strong impact on the $\mathrm{W}$ boson mass.

To summarize: the experimental values of $m_{W}$ and $m_{H_{1}}$ impose stringent and nontrivial constraints on the model parameters. Nevertheless it is easy to identify regions in the parameter space which accommodate the observed values. These regions are characterized by at least one large $\lambda / \Lambda$ coupling and high scalar masses to reduce the tree level reduction of $m_{H_{1}}$ and minimize the $T$ parameter, as well as relatively low higgsino and gaugino Dirac masses to minimize tree level reduction.

The proposed benchmark points contain many of the new states within the kinematical reach at the LHC, in particular supersymmetric fermions. Given the Dirac nature of gauginos, together with R-charge conservation, distinctly different signatures in comparison to the MSSM are expected. This motivates further dedicated studies of phenomenological implications of our benchmark points for LHC physics.

\section{Acknowledgments}

P.D. and W.K. would like to thank Florian Staub for continuous help on SARAH and Alex Voigt for help in cross-checking results with FlexibleSUSY. P.D. thanks Gregor Hellwig for providing his STU package for use. We also thank Tim Stefaniak for help concerning HiggsBounds and HiggsSignals, and Tania Robens for useful discussions and comments.

This work was supported in part by the Polish National Science Centre grants under OPUS-2012/05/B/ST2/03306, DEC-2012/05/B/ST2/02597, DEC2011/01/M/ST2/02466 and the German DAAD PPP Poland Project 56269947 "Dark Matter at Colliders", the German DFG Research Training Group 1504 and the DFG grant STO 876/4-1, and the European Commission through the contract PITN-GA-2012-316704 (HIGGSTOOLS).

\section{A Feynman rules}

In this appendix we present Feynman rules for vertices needed in appendix B, which are peculiar for the MRSSM model due to the Dirac nature of neutralinos and different composition of charginos. 

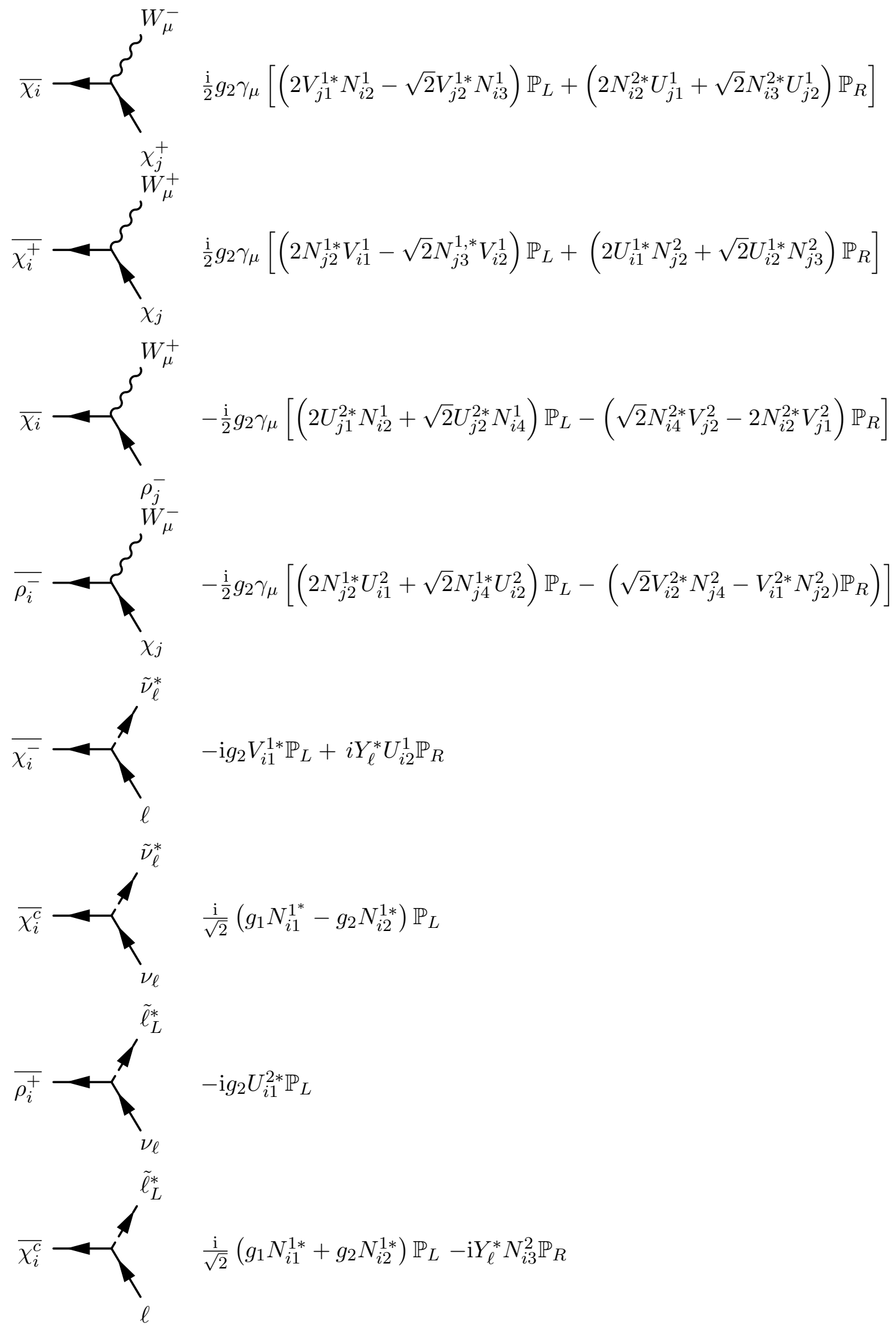


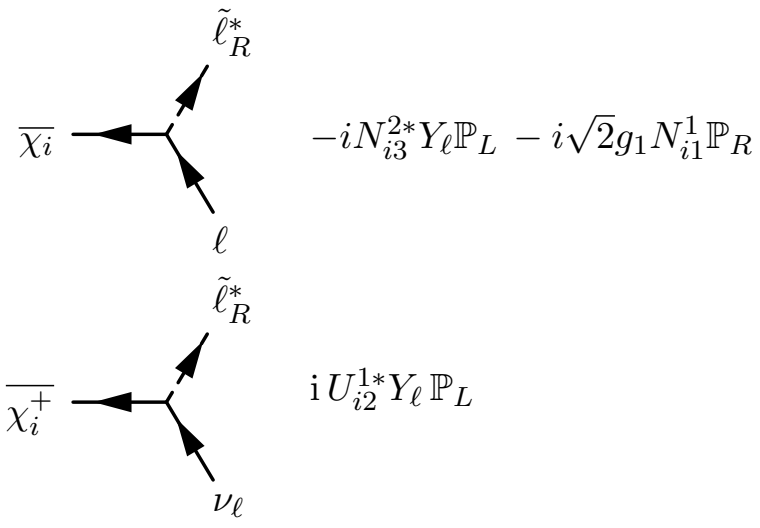

where $\mathbb{P}_{L}=\left(1-\gamma_{5}\right) / 2$ and $\mathbb{P}_{R}=\left(1+\gamma_{5}\right) / 2$. The couplings to quarks/squarks can easily be reproduced by simple substitutions. For simplicity the above rules assume no intergeneration mixing of sfermions.

\section{B Calculation of vertex and box contributions to muon decay}

Here we give an explicit formula for the MRSSM-specific contributions to $\delta_{V B}$

$$
\delta_{V B}=2 \cdot \frac{\sqrt{2}}{g_{2}} \delta V+\frac{2 m_{W}^{2}}{g_{2}^{2}} B+\frac{1}{2} \delta Z_{L}^{e}+\frac{1}{2} \delta Z_{L}^{\mu}+\frac{1}{2} \delta Z_{L}^{\nu_{e}}+\frac{1}{2} \delta Z_{L}^{\nu_{\mu}},
$$

where $\delta V$ and $B$ are vertex- and box-corrections for muon decay and $\delta Z_{L}^{i}$ stands for the external wave function renormalization of the lepton $i$.

- External wave functions renormalization

A generic diagram contributing to the renormalization of the external wave functions is shown in figure 6. Decomposing the general fermion self-energy $-\mathrm{i} \Sigma_{f f}$ as

$$
\Sigma_{f f}=\Sigma_{f f}^{L} \not p P_{L}+\Sigma_{f f}^{R} \not p P_{R}+\Sigma_{f f}^{M} M_{f},
$$

one finds, in the flavor conserving limit and assuming that leptons are massless, for the left-handed projector

$$
\begin{aligned}
\Sigma_{\mu \mu}^{L, \text {MRSSM-SM }}= & \frac{g_{2}^{2}}{16 \pi^{2}} \sum_{i=1}^{2}\left|V_{i 1}^{1}\right|^{2} B_{1}\left(m_{\chi_{i}^{ \pm}}^{2}, m_{\tilde{\nu}_{\mu}}^{2}\right) \\
& +\frac{1}{16 \pi^{2}} \sum_{i=1}^{4}\left|\frac{g_{2} N_{i 2}^{1}+g_{1} N_{i 1}^{1}}{\sqrt{2}}\right|^{2} B_{1}\left(m_{\chi_{i}}^{2}, m_{\tilde{\mu}_{L}}^{2}\right), \\
\Sigma_{\nu \mu \nu}^{L, \text {MRSSM}-S M}= & \frac{g_{2}^{2}}{16 \pi^{2}} \sum_{i=1}^{2}\left|U_{i 1}^{2}\right|^{2} B_{1}\left(m_{\rho_{i}^{ \pm}}^{2}, m_{\tilde{\mu}_{L}}^{2}\right) \\
& +\frac{1}{16 \pi^{2}} \sum_{i=1}^{4}\left|\frac{g_{2} N_{i 2}^{1}-g_{1} N_{i 1}^{1}}{\sqrt{2}}\right|^{2} B_{1}\left(m_{\chi_{i}}^{2}, m_{\tilde{\nu}_{\mu}}^{2}\right),
\end{aligned}
$$




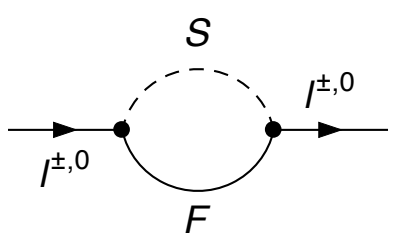

Figure 6. Generic form of a non SM-like contribution to the external wave function renormalization.

where $B_{1}$ is defined according to the LoopTools [58] convention. Because we neglected masses of leptons, the same equations hold for leptons of the first generation. External wave functions are renormalized in the on-shell scheme, i.e.

$$
\delta Z_{i}^{L}=\Sigma_{i i}^{L, M R S S M}
$$

- Vertex correction

Figure 7 contains non-SM corrections to the muon decay vertex. The corresponding analytic expression for the amplitude reads

$$
\begin{aligned}
-\mathrm{i} \delta V= & -\frac{\mathrm{i} g_{2}}{16 \sqrt{2} \pi^{2}} \sum_{i=1}^{2} \sum_{j=1}^{4} g_{2} V_{i 1}^{1 *}\left(\frac{g_{2} N_{j 2}^{1}-g_{1} N_{j 1}^{1}}{\sqrt{2}}\right)\left\{\left(\sqrt{2} N_{j 2}^{1 *} V_{i 1}^{1}-N_{j 3}^{1 *} V_{i 2}^{1}\right)\right. \\
& {\left[m_{\tilde{\nu}_{\mu}}^{2} C_{0}\left(m_{\chi_{i}^{ \pm}}^{2}, m_{\chi_{j}}^{2}, m_{\tilde{\nu}_{\mu}}^{2}\right)+B_{0}\left(m_{\chi_{i}^{ \pm}}^{2}, m_{\chi_{j}}^{2}\right)-2 C_{00}\left(m_{\chi_{i}^{ \pm}}^{2}, m_{\chi_{j}}^{2}, m_{\tilde{\nu}_{\mu}}^{2}\right)\right] } \\
& \left.-m_{\chi_{i}^{ \pm}}^{2} m_{\chi_{j}}^{2}\left(\sqrt{2} N_{j 2}^{2} U_{i 1}^{1 *}+N_{j 3}^{2} U_{i 2}^{1 *}\right) C_{0}\left(m_{\chi_{i}^{ \pm}}^{2}, m_{\chi_{j}}^{2}, m_{\tilde{\nu}_{\mu}}^{2}\right)\right\} \\
& -\frac{\mathrm{i} g_{2}}{16 \sqrt{2} \pi^{2}} \sum_{i=1}^{2} \sum_{j=1}^{4} g_{2} U_{i 1}^{2}\left(\frac{g_{2} N_{j 2}^{1 *}+g_{1} N_{j 1}^{1 *}}{\sqrt{2}}\right)\left\{\left(\sqrt{2} N_{j 2}^{1} U_{i 1}^{2 *}+N_{j 3}^{1} U_{i 2}^{2 *}\right)\right. \\
& {\left[m_{\tilde{\mu}}^{2} C_{0}\left(m_{\rho_{i}^{ \pm}}^{2}, m_{\chi_{j}}^{2}, m_{\tilde{\mu}}^{2}\right)+B_{0}\left(m_{\rho_{i}^{ \pm}}^{2}, m_{\chi_{j}}^{2}\right)-2 C_{00}\left(m_{\rho_{i}^{ \pm}}^{2}, m_{\chi_{j}}^{2}, m_{\tilde{\mu}}^{2}\right)\right] } \\
& \left.\left.-m_{\rho_{i}^{ \pm}}^{2} m_{\chi_{j}}^{2}\left(\sqrt{2} N_{j 2}^{2} U_{i 1}^{1 *}-N_{j 4}^{2 *} V_{i 2}^{2}\right)\right] C_{0}\left(m_{\rho_{i}^{ \pm}}^{2}, m_{\chi_{j}}^{2}, m_{\tilde{\mu}}^{2}\right)\right\} \\
& +\frac{i g_{2}}{8 \sqrt{2} \pi^{2}} \sum_{i=1}^{4}\left(\frac{g_{2} N_{j 2}^{1}-g_{1} N_{j 1}^{1}}{\sqrt{2}}\right)\left(\frac{g_{2} N_{j 2}^{1 *}+g_{1} N_{j 1}^{1 *}}{\sqrt{2}}\right) C_{00}\left(m_{\chi_{i}}^{2}, m_{\tilde{\mu}}^{2}, m_{\tilde{\nu}_{\mu}}^{2}\right),
\end{aligned}
$$

where all Passarino-Veltman's functions follow, as before, the LoopTools convention. Although diagrams from figure 7 have the same analytic expression as in the MSSM, due to the absorption of $\tilde{W}^{ \pm}$into $\chi^{+}$and $\rho^{-}$, respectively, diagram 2 is in principle independent in magnitude from diagram 1 , as it depends on the mass of a different particle. 

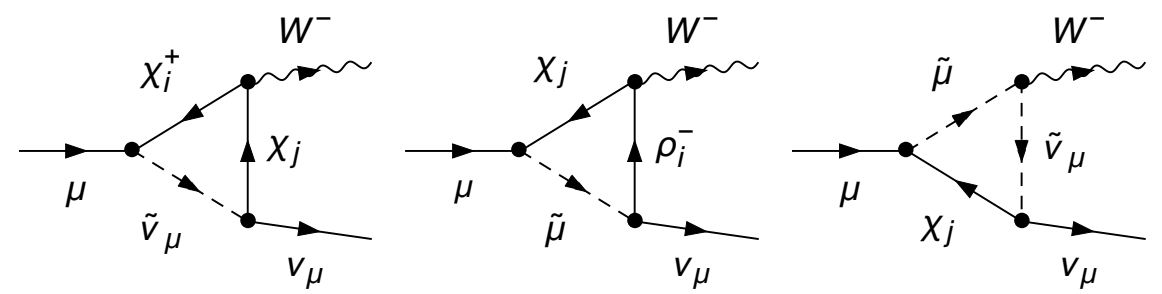

Figure 7. Non-SM corrections to $\mu^{-} \rightarrow W^{-} \nu_{\mu}$ decay vertex. Diagrams proportional to the muon Yukawa are not shown.
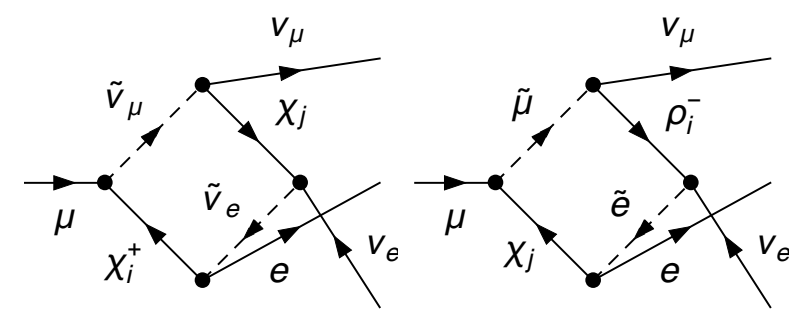

Figure 8. Non-SM box contributions to $\mu^{-} \rightarrow \nu_{\mu} e^{-} \bar{\nu}_{e}$ in the MRSSM. Diagrams proportional to lepton's Yukawa or vanishing in the flavor-conserving limit are not shown.

- Box correction

Figure 8 contains the most relevant box-type contributions to muon decay in the MRSSM. Those diagrams are both UV and IR finite. The expression for them, after factorizing out the spinor structure (cf. eq. (B.1)), reads

$$
\begin{aligned}
-\mathrm{i} B= & -\frac{\mathrm{i}}{16 \pi^{2}} \sum_{i=1}^{2} \sum_{j=1}^{4}\left|g_{2}^{2} V_{i 1}^{1}\left(\frac{g_{1} N_{j 1}^{1}-g_{2} N_{j 2}^{1}}{\sqrt{2}}\right)\right|^{2} D_{00}\left(m_{\chi_{i}^{ \pm}}^{2}, m_{\chi_{j}}^{2}, m_{\tilde{\nu}_{\mu}}^{2}, m_{\tilde{\nu}_{e}}^{2}\right) \\
& -\frac{\mathrm{i}}{16 \pi^{2}} \sum_{i=1}^{2} \sum_{j=1}^{4}\left|g_{2}^{2} U_{i 1}^{2}\left(\frac{g_{1} N_{j 1}^{1}+g_{2} N_{j 2}^{1}}{\sqrt{2}}\right)\right|^{2} D_{00}\left(m_{\rho_{i}^{ \pm}}^{2}, m_{\chi_{j}}^{2}, m_{\tilde{\mu}}^{2}, m_{\tilde{e}}^{2}\right),
\end{aligned}
$$

where $D_{00}$ is defined according to the LoopTools convention. The structure of this correction is different from the one in the MSSM due to the Dirac nature of MRSSM's neutralinos and the fact that $\tilde{W}^{+}$and $\tilde{W}^{-}$are parts of two different types of charginos. This forbids the existence of two additional MSSM-like diagrams with mass-term induced $\tilde{W}^{+}-\tilde{W}^{-}$. Although distinct from the MSSM, for benchmark points under consideration, the contribution from the box-correction is below $1 \mathrm{MeV}$.

In total, for the benchmark points considered, the $\delta_{V B}$ accounts for about $150-$ $175 \mathrm{MeV}$ downward shift on $m_{W}$, which comprises $-350 \mathrm{MeV}$ pure SM correction and $+175 \mathrm{MeV}$ MRSSM part. It should be recalled that $\delta_{V B}$ is not finite nor gaugeindependent and as such these numbers should be interpreted with care. Also, the parameter dependence of $\delta_{V B}$ is mild and it generates mostly a constant shift in $m_{W}$. 
Open Access. This article is distributed under the terms of the Creative Commons Attribution License (CC-BY 4.0), which permits any use, distribution and reproduction in any medium, provided the original author(s) and source are credited.

\section{References}

[1] R. Haag, J.T. Lopuszanski and M. Sohnius, All Possible Generators of Supersymmetries of the s Matrix, Nucl. Phys. B 88 (1975) 257 [inSPIRE].

[2] A. Salam and J.A. Strathdee, Supersymmetry and Fermion Number Conservation, Nucl. Phys. B 87 (1975) 85 [inSPIRE].

[3] P. Fayet, Supergauge Invariant Extension of the Higgs Mechanism and a Model for the electron and Its Neutrino, Nucl. Phys. B 90 (1975) 104 [INSPIRE].

[4] P. Fayet, $N=2$ Extended Supersymmetric GUTs: Gauge Boson/Higgs Boson Unification, Mass Spectrum and Central Charges, Nucl. Phys. B 246 (1984) 89 [inSPIRE].

[5] P. Fayet, Six-dimensional Supersymmetric QED, $R$ Invariance and $N=2$ Supersymmetry Breaking by Dimensional Reduction, Nucl. Phys. B 263 (1986) 649 [inSPIRE].

[6] S. Abel and M. Goodsell, Easy Dirac Gauginos, JHEP 06 (2011) 064 [arXiv:1102.0014] [INSPIRE].

[7] K. Benakli, Dirac Gauginos: A User Manual, Fortsch. Phys. 59 (2011) 1079 [arXiv:1106.1649] [INSPIRE].

[8] P.J. Fox, A.E. Nelson and N. Weiner, Dirac gaugino masses and supersoft supersymmetry breaking, JHEP 08 (2002) 035 [hep-ph/0206096] [INSPIRE].

[9] G.D. Kribs and A. Martin, Dirac Gauginos in Supersymmetry - Suppressed Jets + MET Signals: A Snowmass Whitepaper, arXiv:1308.3468 [INSPIRE].

[10] I. Jack and D.R.T. Jones, Nonstandard soft supersymmetry breaking, Phys. Lett. B 457 (1999) 101 [hep-ph/9903365] [INSPIRE].

[11] M.D. Goodsell, Two-loop RGEs with Dirac gaugino masses, JHEP 01 (2013) 066 [arXiv: 1206.6697] [INSPIRE].

[12] K. Benakli and M.D. Goodsell, Dirac Gauginos in General Gauge Mediation, Nucl. Phys. B 816 (2009) 185 [arXiv:0811.4409] [INSPIRE].

[13] K. Benakli and M.D. Goodsell, Dirac Gauginos, Gauge Mediation and Unification, Nucl. Phys. B 840 (2010) 1 [arXiv: 1003.4957] [INSPIRE].

[14] G.D. Kribs, E. Poppitz and N. Weiner, Flavor in supersymmetry with an extended R-symmetry, Phys. Rev. D 78 (2008) 055010 [arXiv:0712.2039] [INSPIRE].

[15] C. Frugiuele and T. Gregoire, Making the Sneutrino a Higgs with a $U(1)_{R}$ Lepton Number, Phys. Rev. D 85 (2012) 015016 [arXiv:1107.4634] [InSPIRE].

[16] C. Frugiuele, T. Gregoire, P. Kumar and E. Ponton, ' $L=R^{\prime}-\mathrm{U}(1)_{R}$ Lepton Number at the LHC, JHEP 05 (2013) 012 [arXiv:1210.5257] [INSPIRE].

[17] R. Davies, J. March-Russell and M. McCullough, A Supersymmetric One Higgs Doublet Model, JHEP 04 (2011) 108 [arXiv:1103.1647] [INSPIRE].

[18] F. Riva, C. Biggio and A. Pomarol, Is the 125 GeV Higgs the superpartner of a neutrino?, JHEP 02 (2013) 081 [arXiv: 1211.4526] [InSPIRE]. 
[19] S. Chakraborty and S. Roy, Higgs boson mass, neutrino masses and mixing and keV dark matter in an $\mathrm{U}(1)_{R}-$ lepton number model, JHEP 01 (2014) 101 [arXiv:1309.6538] [INSPIRE].

[20] R. Fok and G.D. Kribs, $\mu \rightarrow e$ in R-symmetric Supersymmetry, Phys. Rev. D 82 (2010) 035010 [arXiv: 1004.0556] [INSPIRE].

[21] G.D. Kribs and A. Martin, Supersoft Supersymmetry is Super-Safe, Phys. Rev. D 85 (2012) 115014 [arXiv: 1203.4821] [inSPIRE].

[22] M.R. Buckley, D. Hooper and J. Kumar, Phenomenology of Dirac Neutralino Dark Matter, Phys. Rev. D 88 (2013) 063532 [arXiv:1307.3561] [InSPIRE].

[23] E.J. Chun, J.-C. Park and S. Scopel, Dirac gaugino as leptophilic dark matter, JCAP 02 (2010) 015 [arXiv: 0911.5273] [INSPIRE].

[24] G. Bélanger, K. Benakli, M. Goodsell, C. Moura and A. Pukhov, Dark Matter with Dirac and Majorana Gaugino Masses, JCAP 08 (2009) 027 [arXiv:0905.1043] [INSPIRE].

[25] S.Y. Choi et al., Color-octet scalars at the LHC, Acta Phys. Polon. B 40 (2009) 1947 [arXiv: 0902.4706] [INSPIRE].

[26] S.Y. Choi, J. Kalinowski, J.M. Kim and E. Popenda, Scalar gluons and Dirac gluinos at the LHC, Acta Phys. Polon. B 40 (2009) 2913 [arXiv:0911.1951] [inSPIRE].

[27] S.Y. Choi et al., Dirac Neutralinos and Electroweak Scalar Bosons of $N=1 / N=2$ Hybrid Supersymmetry at Colliders, JHEP 08 (2010) 025 [arXiv: 1005.0818] [INSPIRE].

[28] S.Y. Choi, D. Choudhury, A. Freitas, J. Kalinowski and P.M. Zerwas, The Extended Higgs System in R-symmetric Supersymmetry Theories, Phys. Lett. B 697 (2011) 215 [Erratum ibid. B 698 (2011) 457] [arXiv: 1012.2688] [INSPIRE].

[29] J. Kalinowski, Exploring Dirac neutralinos and $E W$ adjoint scalars of $N=1 / N=2$ hybrid SUSY at colliders, PoS(ICHEP 2010) 396 [arXiv: 1012.0922] [INSPIRE].

[30] J. Kalinowski, Phenomenology of R-symmetric supersymmetry, Acta Phys. Polon. B 42 (2011) 2425 [INSPIRE].

[31] W. Kotlarski and J. Kalinowski, Scalar gluons at the LHC, Acta Phys. Polon. B 42 (2011) 2485 [INSPIRE].

[32] W. Kotlarski, A. Kalinowski and J. Kalinowski, Searching for Sgluons in the Same-sign Leptons Final State at the LHC, Acta Phys. Polon. B 44 (2013) 2149 [InSPIRE].

[33] K. Benakli, M.D. Goodsell and F. Staub, Dirac Gauginos and the 125 GeV Higgs, JHEP 06 (2013) 073 [arXiv: 1211.0552] [INSPIRE].

[34] E. Bertuzzo, C. Frugiuele, T. Gregoire and E. Ponton, Dirac gauginos, $R$ symmetry and the $125 \mathrm{GeV}$ Higgs, arXiv: 1402.5432 [inSPIRE].

[35] S. Weinberg, Baryon and Lepton Nonconserving Processes, Phys. Rev. Lett. 43 (1979) 1566 [INSPIRE].

[36] N. Sakai and T. Yanagida, Proton Decay in a Class of Supersymmetric Grand Unified Models, Nucl. Phys. B 197 (1982) 533 [INSPIRE].

[37] J.A. Aguilar-Saavedra et al., Supersymmetry parameter analysis: SPA convention and project, Eur. Phys. J. C 46 (2006) 43 [hep-ph/0511344] [INSPIRE].

[38] Particle Data Group collaboration, K.A. Olive et al., Review of Particle Physics (RPP), Chin. Phys. C 38 (2014) 090001 [inSPIRE]. 
[39] Wolfram Research Inc., Mathematica Version 9.0/10.0, Champaign U.S.A. (2012/2014).

[40] F. Staub, Sarah, arXiv:0806.0538 [INSPIRE].

[41] F. Staub, From Superpotential to Model Files for FeynArts and CalcHep/CompHEP, Comput. Phys. Commun. 181 (2010) 1077 [arXiv:0909. 2863] [INSPIRE].

[42] F. Staub, Automatic Calculation of supersymmetric Renormalization Group Equations and Self Energies, Comput. Phys. Commun. 182 (2011) 808 [arXiv: 1002.0840] [inSPIRE].

[43] F. Staub, SARAH 3.2: Dirac Gauginos, UFO output and more, Computer Physics Communications 184 (2013) pp. 1792-1809 [arXiv: 1207.0906] [INSPIRE].

[44] F. Staub, SARAH 4: A tool for (not only SUSY) model builders, Comput. Phys. Commun. 185 (2014) 1773 [arXiv: 1309.7223] [INSPIRE].

[45] W. Porod, SPheno, a program for calculating supersymmetric spectra, SUSY particle decays and SUSY particle production at $e^{+} e^{-}$colliders, Comput. Phys. Commun. 153 (2003) 275 [hep-ph/0301101] [INSPIRE].

[46] W. Porod and F. Staub, SPheno 3.1: Extensions including flavour, CP-phases and models beyond the MSSM, Comput. Phys. Commun. 183 (2012) 2458 [arXiv:1104.1573] [INSPIRE].

[47] P. Athron, J.-h. Park, D. Stöckinger and A. Voigt, FlexibleSUSY - A spectrum generator generator for supersymmetric models, arXiv:1406.2319 [INSPIRE].

[48] B.C. Allanach, SOFTSUSY: a program for calculating supersymmetric spectra, Comput. Phys. Commun. 143 (2002) 305 [hep-ph/0104145] [INSPIRE].

[49] B.C. Allanach, P. Athron, L.C. Tunstall, A. Voigt and A.G. Williams, Next-to-Minimal SOFTSUSY, Comput. Phys. Commun. 185 (2014) 2322 [arXiv:1311.7659] [InSPIRE].

[50] H.E. Haber and R. Hempfling, Can the mass of the lightest Higgs boson of the minimal supersymmetric model be larger than m(Z)?, Phys. Rev. Lett. 66 (1991) 1815 [INSPIRE].

[51] J.R. Ellis, G. Ridolfi and F. Zwirner, On radiative corrections to supersymmetric Higgs boson masses and their implications for LEP searches, Phys. Lett. B 262 (1991) 477 [INSPIRE].

[52] P.H. Chankowski, S. Pokorski and J. Rosiek, Charged and neutral supersymmetric Higgs boson masses: Complete one loop analysis, Phys. Lett. B 274 (1992) 191 [INSPIRE].

[53] M. Sperling, D. Stöckinger and A. Voigt, Renormalization of vacuum expectation values in spontaneously broken gauge theories, JHEP 07 (2013) 132 [arXiv:1305.1548] [INSPIRE].

[54] M. Sperling, D. Stöckinger and A. Voigt, Renormalization of vacuum expectation values in spontaneously broken gauge theories: Two-loop results, JHEP 01 (2014) 068 [arXiv:1310.7629] [INSPIRE].

[55] A.V. Bednyakov, A.F. Pikelner and V.N. Velizhanin, Three-loop Higgs self-coupling $\beta$-function in the Standard Model with complex Yukawa matrices, Nucl. Phys. B 879 (2014) 256 [arXiv: 1310.3806] [INSPIRE].

[56] T. Hahn, Generating Feynman diagrams and amplitudes with FeynArts 3, Comput. Phys. Commun. 140 (2001) 418 [hep-ph/0012260] [INSPIRE].

[57] B. Chokoufe Nejad, T. Hahn, J.-N. Lang and E. Mirabella, FormCalc 8: Better Algebra and Vectorization, J. Phys. Conf. Ser. 523 (2014) 012050 [arXiv: 1310. 0274] [InSPIRE].

[58] T. Hahn and M. Pérez-Victoria, Automatized one loop calculations in four-dimensions and D-dimensions, Comput. Phys. Commun. 118 (1999) 153 [hep-ph/9807565] [INSPIRE]. 
[59] S.R. Coleman and E.J. Weinberg, Radiative Corrections as the Origin of Spontaneous Symmetry Breaking, Phys. Rev. D 7 (1973) 1888 [INSPIRE].

[60] B.C. Allanach, A. Djouadi, J.L. Kneur, W. Porod and P. Slavich, Precise determination of the neutral Higgs boson masses in the MSSM, JHEP 09 (2004) 044 [hep-ph/0406166] [INSPIRE].

[61] G. Degrassi, S. Fanchiotti and A. Sirlin, Relations Between the On-shell and Ms Frameworks and the $M(W)-M(Z)$ Interdependence, Nucl. Phys. B 351 (1991) 49 [InSPIRE].

[62] T. Blank and W. Hollik, Precision observables in $\mathrm{SU}(2) \times \mathrm{U}(1)$ models with an additional Higgs triplet, Nucl. Phys. B 514 (1998) 113 [hep-ph/9703392] [INSPIRE].

[63] P.H. Chankowski, S. Pokorski and J. Wagner, (Non)decoupling of the Higgs triplet effects, Eur. Phys. J. C 50 (2007) 919 [hep-ph/0605302] [InSPIRE].

[64] D. Lopez-Val and T. Robens, Delta $r$ and the W-boson mass in the Singlet Extension of the Standard Model, arXiv:1406.1043 [INSPIRE].

[65] S. Fanchiotti, B.A. Kniehl and A. Sirlin, Incorporation of QCD effects in basic corrections of the electroweak theory, Phys. Rev. D 48 (1993) 307 [hep-ph/9212285] [InSPIRE].

[66] D.M. Pierce, J.A. Bagger, K.T. Matchev and R.-j. Zhang, Precision corrections in the minimal supersymmetric standard model, Nucl. Phys. B 491 (1997) 3 [hep-ph/9606211] [INSPIRE].

[67] M.E. Peskin and T. Takeuchi, A New constraint on a strongly interacting Higgs sector, Phys. Rev. Lett. 65 (1990) 964 [INSPIRE].

[68] W.J. Marciano and J.L. Rosner, Atomic parity violation as a probe of new physics, Phys. Rev. Lett. 65 (1990) 2963 [Erratum ibid. 68 (1992) 898] [INSPIRE].

[69] M.E. Peskin and T. Takeuchi, Estimation of oblique electroweak corrections, Phys. Rev. D 46 (1992) 381 [INSPIRE].

[70] D.C. Kennedy and P. Langacker, Precision electroweak experiments and heavy physics: A Global analysis, Phys. Rev. Lett. 65 (1990) 2967 [Erratum ibid. 66 (1991) 395] [INSPIRE].

[71] D.C. Kennedy and P. Langacker, Precision electroweak experiments and heavy physics: An Update, Phys. Rev. D 44 (1991) 1591 [inSPIRE].

[72] G. Altarelli and R. Barbieri, Vacuum polarization effects of new physics on electroweak processes, Phys. Lett. B 253 (1991) 161 [InSPIRE].

[73] G. Cynolter and E. Lendvai, Electroweak Precision Constraints on Vector-like Fermions, Eur. Phys. J. C 58 (2008) 463 [arXiv:0804.4080] [inSPIRE].

[74] M. Drees and K. Hagiwara, Supersymmetric Contribution to the Electroweak $\rho$ Parameter, Phys. Rev. D 42 (1990) 1709 [inSPIRE].

[75] A. Buckley, PySLHA: a Pythonic interface to SUSY Les Houches Accord data, arXiv: 1305.4194 [INSPIRE].

[76] M. Awramik, M. Czakon, A. Freitas and G. Weiglein, Precise prediction for the $W$ boson mass in the standard model, Phys. Rev. D 69 (2004) 053006 [hep-ph/0311148] [INSPIRE].

[77] A. Ferroglia and A. Sirlin, Comparison of the Standard Theory Predictions of $M_{W}$ and $\sin f^{2}$ $\theta_{\text {eff }}^{\text {lept }}$ with their Experimental Values, Phys. Rev. D 87 (2013) 037501 [arXiv:1211.1864] [INSPIRE]. 
[78] P. Bechtle, O. Brein, S. Heinemeyer, G. Weiglein and K.E. Williams, HiggsBounds: Confronting Arbitrary Higgs Sectors with Exclusion Bounds from LEP and the Tevatron, Comput. Phys. Commun. 181 (2010) 138 [arXiv:0811.4169] [InSPIRE].

[79] P. Bechtle, O. Brein, S. Heinemeyer, G. Weiglein and K.E. Williams, HiggsBounds 2.0.0: Confronting Neutral and Charged Higgs Sector Predictions with Exclusion Bounds from LEP and the Tevatron, Comput. Phys. Commun. 182 (2011) 2605 [arXiv:1102.1898] [InSPIRE].

[80] P. Bechtle et al., Recent Developments in HiggsBounds and a Preview of HiggsSignals, PoS (CHARGED 2012) 024.

[81] P. Bechtle et al., HiggsBounds - 4: Improved Tests of Extended Higgs Sectors against Exclusion Bounds from LEP, the Tevatron and the LHC, Eur. Phys. J. C 74 (2014) 2693 [arXiv: 1311.0055] [INSPIRE].

[82] P. Bechtle, S. Heinemeyer, O. Stål, T. Stefaniak and G. Weiglein, HiggsSignals: Confronting arbitrary Higgs sectors with measurements at the Tevatron and the LHC, Eur. Phys. J. C 74 (2014) 2711 [arXiv:1305.1933] [InSPIRE].

[83] O. Stål and T. Stefaniak, Constraining extended Higgs sectors with HiggsSignals, PoS (EPS-HEP 2013) 314 [arXiv: 1310.4039] [INSPIRE].

[84] J.E. Camargo-Molina, B. O'Leary, W. Porod and F. Staub, Vevacious: A Tool For Finding The Global Minima Of One-Loop Effective Potentials With Many Scalars, Eur. Phys. J. C 73 (2013) 2588 [arXiv:1307.1477] [INSPIRE].

[85] J.A. Casas, A. Lleyda and C. Muñoz, Strong constraints on the parameter space of the MSSM from charge and color breaking minima, Nucl. Phys. B 471 (1996) 3 [hep-ph/9507294] [INSPIRE]. 